\title{
Numerically-Based Parametric Analysis of Plain Fin and Tube Compact Heat Exchangers
}

\author{
Jeanette Cobian-Iñiguez ${ }^{a}$, Angela $\mathrm{Wu}^{a}$, Florian Dugast ${ }^{a, b}$, Arturo Pacheco-Vega ${ }^{a 1}$ \\ ${ }^{a}$ Department of Mechanical Engineering \\ California State University, Los Angeles \\ Los Angeles, CA 90032, USA. \\ ${ }^{b}$ Department of Mechanical Engineering \\ Polytech' Nantes École D'Ingénieurs, Université de Nantes \\ 44306 Nantes Cedex 3, France.
}

March 27, 2015

\begin{abstract}
In the present study, the hydrodynamic and heat transfer characteristics of six-tube-row compact fin and tube heat exchangers have been investigated numerically by introducing a methodology of analysis based on local and global energy balances, from three-dimensional velocity and temperature fields. The aim is to analyze the influence of operating conditions and the geometry to design more efficient devices. Tube diameter, fin spacing and tube layout are the geometrical parameters; the over-tube fluid velocity, via Reynolds number, is used as the parameter of operation. Using the procedure, along with the concept of fraction of the total heat rate available for the specific device, the results have shown that, fluid velocity plays an important role, whereas the role of tube layout is minor, and that the effects of tube diameter and fin spacing being closely related to the magnitude of the fluid velocity. For small velocities, $99 \%$ of the heat rate that could be potentially achieved occurs closer to the inlet of the device, whereas for large velocity-values, nearly the entire length is necessary. In addition, the influence of the tube-diameter on both heat transfer and pressure drop is negligible at small velocities but progressively increases at bigger velocity-values; a similar -but less pronounced- effect is produced by fin spacing, where the influence of tubediameter is relevant only for larger fin spacings. The approach introduced here is useful in providing, at the expense of some generality, high accuracy and clear information on the thermal convection process in these devices, since intermediate steps via Nusselt numbers and heat transfer coefficients are not needed.
\end{abstract}

Keywords: Heat exchanger; Numerical analysis; Parametric study; Fluid flow; Heat transfer.

\footnotetext{
${ }^{1}$ Corresponding author, Tel.: (323) 343-4492, Fax: (323) 343-5004, e-mail: apacheco@calstatela.edu
} 


\section{NOMENCLATURE}

\begin{tabular}{|c|c|}
\hline$A$ & cross sectional area $\left[\mathrm{m}^{2}\right]$ \\
\hline$c_{p}$ & specific heat at constant pressure $[\mathrm{J} / \mathrm{kg} \cdot \mathrm{K}]$ \\
\hline$D$ & tube diameter $[\mathrm{mm}]$ \\
\hline$H$ & height of heat exchanger $[\mathrm{m}]$ \\
\hline$k$ & thermal conductivity of fluid $[\mathrm{W} / \mathrm{m} \cdot \mathrm{K}]$ \\
\hline$L$ & length of heat exchanger $[\mathrm{m}]$ \\
\hline$L_{L}$ & center-tube distance to leading edge $[\mathrm{m}]$ \\
\hline$L_{T}$ & center-tube distance to trailing edge $[\mathrm{m}]$ \\
\hline$L_{x 1}$ & extended computational domain ahead of fins $[\mathrm{m}]$ \\
\hline$L_{x 2}$ & extended computational domain after fins $[\mathrm{m}]$ \\
\hline$\Delta L$ & length of fin section $[\mathrm{m}]$ \\
\hline$N_{T}$ & number of tubes \\
\hline$N u$ & Nusselt number \\
\hline $\mathbf{n}$ & unit vector \\
\hline$P_{l}$ & longitudinal tube pitch $[\mathrm{m}]$ \\
\hline$P_{t}$ & transverse tube pitch $[\mathrm{m}]$ \\
\hline$p$ & pressure $[\mathrm{Pa}]$ \\
\hline$\Delta p$ & pressure drop $[\mathrm{Pa}]$ \\
\hline$Q$ & heat transfer rate $[\mathrm{W}]$ \\
\hline$Q_{L_{x 1}}$ & heat transfer rate in section $L_{x 1}[\mathrm{~W}]$ \\
\hline$Q_{T}$ & total heat transfer rate $[\mathrm{W}]$ \\
\hline$R e_{D}$ & Reynolds number based on tube diameter \\
\hline$R e_{\delta}$ & Reynolds number based on fin spacing \\
\hline$T$ & fluid temperature $[\mathrm{K}]$ \\
\hline$T_{s}$ & surface temperature $[\mathrm{K}]$ \\
\hline $\mathbf{u}$ & Cartesian velocity vector $[\mathrm{m} / \mathrm{s}]$ \\
\hline$u, v, w$ & Cartesian velocities $[\mathrm{m} / \mathrm{s}]$ \\
\hline$u_{\text {in }}$ & inlet frontal velocity $[\mathrm{m} / \mathrm{s}]$ \\
\hline$x$ & Streamwise coordinate $[\mathrm{m}]$ \\
\hline$x, y, z$ & Cartesian coordinates $[\mathrm{m}]$ \\
\hline \multicolumn{2}{|c|}{ Greek symbols } \\
\hline$\delta$ & fin spacing $[\mathrm{mm}]$ \\
\hline$\mu$ & dynamic fluid viscosity $[\mathrm{kg} / \mathrm{m} \cdot \mathrm{s}]$ \\
\hline$\nu$ & kinematic fluid viscosity $\left[\mathrm{m}^{2} / \mathrm{s}\right]$ \\
\hline$\rho$ & fluid density $\left[\mathrm{kg} / \mathrm{m}^{3}\right]$ \\
\hline \multicolumn{2}{|c|}{ Subscripts and superscripts } \\
\hline$b$ & baseline values \\
\hline$\overline{(\cdot)}$ & mean values \\
\hline
\end{tabular}




$\begin{array}{ll}\text { in } & \text { inlet } \\ j & j \text {-th fin section } \\ \text { out } & \text { outlet }\end{array}$




\section{INTRODUCTION}

Compact heat exchangers are widely used in industrial applications since they form an integral part of heating, cooling, ventilation and air conditioning (HVAC), power generation and manufacturing systems. In these thermal devices a common geometry is the plate-fin and tube configuration, in which a liquid is usually driven inside tubes that are exposed to an external flow of a gas, being the fluids at different temperatures. The two typical tube alignments; i.e., in-line and staggered, are illustrated schematically in Figure 1. Thermophysical properties of the gas; e.g., air, and the often laminar nature of the flow in these systems cause the over-tube thermal resistance to critically constrain the transfer of energy. To alleviate the problem the heat transfer area per unit volume is usually very large. Thus, an important design objective for compact heat exchangers is to maximize device compactness while ensuring efficient heat transfer; in this way, operating and manufacturing costs can be significantly reduced while minimizing environmental impact.

Many numerical and experimental studies have aimed at analyzing compact heat exchangers to enhance their performance. Fiebig et al. [1] examined, via Nusselt numbers, the conjugate heat transfer of a finned tube for different Reynolds numbers and fin efficiencies, indicating that a more efficient transfer of energy is obtained in the fin section upstream of the tube. The influence of various geometrical parameters of multi-row heat exchangers was studied by Jang et al. [2], He et al. [3] and Xie et al. [4]. The results from [2] show that the average transfer coefficient and the pressure drop are both larger for staggered arrays than for in-line, whereas all the aforementioned studies report that, when the number of tube-rows is larger than four [2, 4], and three [3] from the field synergy principle viewpoint, its effect on the average transfer coefficient is small. The impact of fin height, tube location and ellipticity of the tube was investigated by Erek et al. [5] for one-tube section of the device under a single operating condition. They observed that by placing the tube at the downstream region of the fin, or increasing ellipticity of the tube, the transfer of heat is enhanced. Abu Madi et al. [6] analyzed the effect of fin-type and number of tubes on the system performance and correlated their experimental results using Colburn $j$ - and friction $f$-factors. A comparable work by Saboya and Saboya [7] was carried out for one- and two-row arrangements of platefin devices using Nusselt numbers and a naphthalene sublimation technique. The influence of flow behavior on heat transfer in a two-row tube configuration was studied by Tsai et al. [8] using topological theory. Their findings show that flow structure, particularly the horseshoe vortex, has a direct effect on the span-averaged Nusselt number and pressure drop. Similar studies to those of [2] and [8] were carried out by Tutar and Akkoca [9] and Zhang et al. [10], 
with emphasis in transient effects and horseshoe vortex formation on Nusselt number and pressure coefficient, reporting a local enhancement of both quantities.

In this study, we perform numerical simulations over plain-fin and tube heat exchangers and explore the heat transfer enhancement in these devices from a different angle; i.e., the increase in the efficacy of the system by identifying the regions in a complete device where most of the heat transfer takes place, and potentially eliminating those that do not contribute significantly. We also propose a methodology of analysis on the basis of local and global energy balances from the field quantities, and use the heat transfer rate instead of the common Nusselt number as the basis for the calculations; it enables higher accuracy [11]-[14] since neither transfer coefficients nor characteristic temperatures are needed. Using an earlier version of the approach, Motamedi et al. [15] examined a fifteen-tube heat exchanger system for constant values of diameter and fin spacing, and showed that it was possible to reduce the size of the device. The governing equations in Cartesian coordinates are first formulated and then solved on a representative computational domain by the finite element method. From the computed velocity, pressure and temperature fields, heat rates and pressure drops -obtained from the above procedure- are then used to analyze the efficacy of the type of tube alignment and the influence of Reynolds number, fin spacing and diameter size. By

using the concept of fraction of the total heat rate available for the specific device, solutions for each case are computed and their streamwise distribution finally determined.

\section{PROBLEM DESCRIPTION AND MATHEMATICAL MODEL}

Consider the in-line and staggered configurations of a plain-fin and tube compact heat exchanger shown in Figure 1. The gas is air, and flows within the laminar regime in the direction of the arrows, cooling a hot fluid; e.g., water, flowing at high velocity inside the tubes; the fins are separated a distance $\delta, L$ is the nominal length and $H$ is its height. Regardless of the type of tube alignment, whether in-line or staggered, the heat exchanger under analysis has nominal dimensions: $L=38 \mathrm{~cm}$ and $H=44 \mathrm{~cm}$, with a number of tube rows $N_{T}=6$. The values of fin spacing and tube diameter, which will be used in the subsequent sections as baseline values for the analysis, are: $\delta_{b} \equiv \delta=4.4 \mathrm{~mm}$ and $D_{b} \equiv D=31.8 \mathrm{~mm}$. Other important dimensions are: longitudinal fin pitch $P_{l}=63.6$ $\mathrm{mm}$, center-tube distances to the leading and trailing edges $L_{L}=31.8 \mathrm{~mm}$ and $L_{T}=31.8$ $\mathrm{mm}$, respectively, and transverse tube pitch $P_{t}=63.6 \mathrm{~mm}$ for in-line and $P_{t}=31.8 \mathrm{~mm}$ for staggered arrangements.

Since the focus of this investigation is on the numerical analysis of the external side of the 
heat exchanger, a schematic of the computational domain for the two configurations, capable of resembling the entire device, is portrayed in Figure 2, with the preceding parameters being also depicted. From the figure it can be observed that although we use the natural symmetry of the system in the $y$ - and $z$-directions (height and width of the system), a major difference from other investigations which concentrate on a single-tube section, here we consider the complete channel length for analysis. In addition, the common periodic conditions in the streamwise direction will not be adopted; instead, we follow $[2,4,16]$ and implement two additional sections, named $L_{x 1}$ and $L_{x 2}$, to accurately enforce the boundary conditions at both inlet and outlet of the computational domain.

Consider the incompressible flow of a Newtonian fluid, with constant properties, in the laminar regime, under steady-state conditions, and without body forces and viscous dissipation. The mathematical model in Cartesian coordinates is given by

$$
\begin{aligned}
\frac{\partial u}{\partial x}+\frac{\partial v}{\partial y}+\frac{\partial w}{\partial z} & =0 \\
u \frac{\partial u}{\partial x}+v \frac{\partial u}{\partial y}+w \frac{\partial u}{\partial z} & =-\frac{1}{\rho} \frac{\partial p}{\partial x}+\nu\left(\frac{\partial^{2} u}{\partial x^{2}}+\frac{\partial^{2} u}{\partial y^{2}}+\frac{\partial^{2} u}{\partial z^{2}}\right), \\
u \frac{\partial v}{\partial x}+v \frac{\partial v}{\partial y}+w \frac{\partial v}{\partial z} & =-\frac{1}{\rho} \frac{\partial p}{\partial y}+\nu\left(\frac{\partial^{2} v}{\partial x^{2}}+\frac{\partial^{2} v}{\partial y^{2}}+\frac{\partial^{2} v}{\partial z^{2}}\right), \\
u \frac{\partial w}{\partial x}+v \frac{\partial w}{\partial y}+w \frac{\partial w}{\partial z} & =-\frac{1}{\rho} \frac{\partial p}{\partial z}+\nu\left(\frac{\partial^{2} w}{\partial x^{2}}+\frac{\partial^{2} w}{\partial y^{2}}+\frac{\partial^{2} w}{\partial z^{2}}\right), \\
u \frac{\partial T}{\partial x}+v \frac{\partial T}{\partial y}+w \frac{\partial T}{\partial z} & =\frac{k}{\rho c_{p}}\left(\frac{\partial^{2} T}{\partial x^{2}}+\frac{\partial^{2} T}{\partial y^{2}}+\frac{\partial^{2} T}{\partial z^{2}}\right),
\end{aligned}
$$

where, $u, v$ and $w$ are the Cartesian velocities in the directions $x, y$ and $z$, respectively; $p$ is the pressure, $\rho$ the density, $\nu=\mu / \rho$ is the kinematic viscosity, $c_{p}$ the specific heat, $k$ its thermal conductivity and $T$ is the air temperature. For air, $\rho=1.193 \mathrm{~kg} / \mathrm{m}^{3}, \nu=1.52 \times 10^{-5}$ $\mathrm{m}^{2} / \mathrm{s}, c_{p}=1007 \mathrm{~J} / \mathrm{kg} \cdot \mathrm{K}$, and $k=0.0257 \mathrm{~W} / \mathrm{K} \cdot \mathrm{m}$.

Based on the minimum sections of Figure 2, where the origin of the coordinate system has been placed at the intersection of the mid-plane and the inlet plane at the leading-edge of the heat exchanger, the boundary conditions are: non-slip impermeable walls, symmetry at the mid-plane $z=0$ and at those constant- $y$ planes where the condition naturally arises; i.e., $y=0$ and $y=P_{t} / 2$ (or $y=P_{t}$ for the staggered case), uniform flow at the inlet and fully developed flow at the outlet, plus uniform inlet temperature, isothermal walls and zero temperature gradients at the outlet. Note that considering a conjugate version of this problem could slightly improve the calculations; however, it has been shown by [17] that the difference between ideal and real fins is usually small, and most computations reported in 
the literature have used the isothermal-wall assumption without significantly degrading the accuracy of the results.

By dividing the computational region in three sections; i.e., domain inlet of length $L_{x 1}$, the fins segment with length $L$ and the outlet domain of length $L_{x 2}$, the boundary conditions are defined mathematically as follows:

- Upstream extended region, $L x_{1}$ :

At the inlet plane $x=-L_{x 1}$ :

$$
u=u_{\text {in }}=\text { const. }, \quad T=T_{\text {in }}=\text { const. }, \quad v=w=0 .
$$

At the lower and upper boundaries $y=0$ and $y=P_{t} / 2\left(y=P_{t}\right.$ for staggered tubes):

$$
\frac{\partial u}{\partial y}=\frac{\partial w}{\partial y}=0, \quad v=0, \quad \frac{\partial T}{\partial y}=0 .
$$

At the mid-plane $z=0$, and front boundary $z=\delta / 2$ :

$$
\frac{\partial u}{\partial z}=\frac{\partial v}{\partial z}=0, \quad w=0, \quad \frac{\partial T}{\partial z}=0 .
$$

- Heat exchanger domain (fins and tubes region), $L$ :

At the fin $z=\delta / 2$, and tube $\left[x-L_{c}+(j-1) \Delta L\right]^{2}+y^{2} \geq D^{2} / 4, j=1, \cdots, N_{T}$, and $N_{T}=6$, surfaces:

$$
u=v=w=0, \quad T=T_{s}=\text { const. }
$$

At the lower and upper boundaries $y=0$ and $y=P_{t} / 2\left(y=P_{t}\right.$ for staggered tubes):

$$
\frac{\partial u}{\partial y}=\frac{\partial w}{\partial y}=0, \quad v=0, \quad \frac{\partial T}{\partial y}=0 .
$$

At the mid-plane $z=0$ :

$$
\frac{\partial u}{\partial z}=\frac{\partial v}{\partial z}=0, \quad w=0, \quad \frac{\partial T}{\partial z}=0 .
$$

- Downstream extended region, $L x_{2}$ :

At the outflow plane $x=L+L_{x 2}$ :

$$
\frac{\partial u}{\partial x}=\frac{\partial v}{\partial x}=\frac{\partial w}{\partial x}=\frac{\partial T}{\partial x}=0
$$


At the lower and upper boundaries $y=0$ and $y=P_{t} / 2\left(y=P_{t}\right.$ for staggered tubes):

$$
\frac{\partial u}{\partial y}=\frac{\partial w}{\partial y}=0, \quad v=0, \quad \frac{\partial T}{\partial y}=0 .
$$

At the mid-plane $z=0$, and front $z=\delta / 2$ boundaries:

$$
\frac{\partial u}{\partial z}=\frac{\partial v}{\partial z}=0, \quad w=0, \quad \frac{\partial T}{\partial z}=0 .
$$

Importantly, the actual values of $L_{x 1}$ and $L_{x 2}$ depend on the operating conditions and the heat tube configuration. However, instead of using fixed values [2, 4], these quantities are computed via an iterative process. Starting with very large values for both quantities, one of them is fixed while the other is decreased. The streamwise velocity at specific locations in the leading edge for $L_{x 1}$ and trailing edge for $L_{x 2}$, is used as control parameter. The minimum length corresponds to the last value for which $u$ is less than a preset tolerance of $2 \%$ with respect to the velocity computed with the initial values of the extended lengths. Once the value of one of the quantities is computed, the process is carried out for the other. For the baseline configuration and $R e_{D}=960$, which corresponds to the upper bound value for the flow, $L_{x 1}=3 D_{b}$ and $L_{x 2}=25 D_{b}$.

\section{NUMERICAL METHODOLOGY}

\subsection{Discretization Technique}

The governing equations (1)-(5) were discretized on the computational domain and solved by the finite element method. Although other numerical techniques, like those based on finite differences have been developed [18], and successfully applied to a number of fluid flow and heat transfer problems [19]-[21], the main advantage of the finite element technique is its treatment of the boundary conditions on curved surfaces.

The problem under analysis was solved using the general-purpose finite-element software COMSOL Multiphysics (http://www.comsol.com). Here, the computational domain is discretized using a three-dimensional mesh; with hexahedral elements being applied in it, and quadrilateral elements along the boundaries. The degrees of freedom for temperature, velocity and pressure are all assigned at the nodes of the elements. To ensure accurate results while maintaining a manageable CPU time, more dense meshing is used near the walls. Schematics of typical discretization domains for the two tube alignments are depicted in Figure 3 where, for each section, 128 elements are evenly distributed around the tube perimeter and mapped 
outward in a radial fashion to the outer edges of the section, and subsequently linked to a regular mapped mesh (e.g. at $L_{x 1}$ and $L_{x 2}$ ). A total of 193,280 elements were used in each tube-arrangement. In all cases, element sizes close to the walls are sufficiently small to be able to resolve the boundary layers. The velocity and temperature fields were computed from the resulting system of algebraic equations by the generalized minimum residual (GMRES) iterative solver, for which the relative tolerance was set to $10^{-6}$.

\subsection{Grid Independence Tests}

To ensure grid independence of the numerical results, several grids were tested using different values of inlet velocity (i.e., Reynolds numbers). These tests were done for pressure $p$, and streamwise velocity $u$, at a fixed point in the domain of each configuration. A typical set at $(63.6,15.9,1.1) \mathrm{mm}$, for an inlet velocity $u_{i n}=0.23 \mathrm{~m} / \mathrm{s}\left(R e_{D}=480\right)$, is illustrated in Figure 4. From the figure, it can be seen that the error in the values of both velocity and pressure with respect to results from the finest mesh decreases exponentially as the mesh is refined, with velocity values achieving convergence before those of the pressure. The figure also illustrates that regardless of the tube arrangement, a grid with at least 193,280 elements is sufficient to achieve an accuracy within $2 \%$ of the results obtained with a grid containing 4.4 times as many elements while maintaining a manageable CPU time.

\subsection{Model Verification}

Reliability of the model, and the methodology of solution, was verified by analyzing two different plate fin and tube heat exchanger systems. The first is a device with a single-row tube layout that was studied experimentally and numerically by Romero-Mendez et al. [16] to investigate the influence of fin spacing on the over-tube heat transfer. Details of the geometrical configuration, the normalization parameters for their governing equations, as well as the numerical results in terms of local and overall Nusselt numbers, dimensionless fin spacing and pressure drop, are all reported in [16] for two values of Reynolds number. Equations (1)-(5), along with the corresponding boundary conditions, were solved for the aforementioned system using $D=31.8 \mathrm{~mm}$ as the basis for the heat exchanger geometry. Following their definitions for the characteristic quantities, the current numerical solutions of overall Nusselt number $N u^{*}$, ratio of Nusselt to fin spacing $N u^{*} / S^{*}$, and dimensionless pressure drop $\Delta p^{*}$, as functions of the dimensionless fin spacing $S^{*}$, are shown in Figure 5 (a), for $R e^{*}=630$, along with those of Romero-Mendez et al. [16]. In the figure, the present computations are portrayed with the symbol " $\diamond$ ", whereas "-" corresponds to the 
numerical simulations reported in [16]. From the figure it is clear that both qualitatively and quantitatively the results from the present model and methodology of solution, agree very well with those of [16]; the absolute value of the percentage difference $E$ for $N u^{*}$ (or $\left.N u^{*} / S^{*}\right)$, and $\Delta p^{*}$, are in the ranges $E_{N u^{*}} \in[0.8,3.5] \%$ and $E_{\Delta p^{*}} \in[4.9,7.9] \%$, respectively, with corresponding average deviations of $2.6 \%$ and $6.8 \%$.

The second test case is that of an experimental apparatus with a three tube-row staggered arrangement that was examined by Kang et al. [22] for a wide range of Reynolds numbers. The schematic of the heat exchanger and its geometrical dimensions, along the corresponding definitions of Reynolds number $R e^{*}$, average Nusselt number $N u^{*}$, and friction factor $f^{*}$, among other quantities, are provided in [22]. In the present work, velocity, pressure and temperature fields were first obtained from solution of Eqs. (1)-(5) and the boundary conditions, and then used to build the final values of both Nusselt number and friction factor. By adopting the definitions of the typical characteristic quantities, including: characteristic temperature difference, heat transfer coefficient, pressure drop and heat transfer surfaces, as reported by Xie et al. [4], and Zhou et al. [23], the numerical values of $N u^{*}$ and $f^{*}$ are illustrated using the symbol " $\diamond$ " in Figure 5(b). Also shown in the figure are the experimental values of Kang et al. [22] "ם" along with their correlation "-.-". Note that although the experiments reported in [22] are in the range for Reynolds number $R e^{*} \in[350,15600]$, we have restricted ourselves to the range $R e^{*} \in[420,1800]$ which is well within the laminar region of the external flow. From the figure it can be seen that the current numerical values for both $N u^{*}$ and $f^{*}$ are in good agreement with the benchmark experiments and the correlations; the relative error for the Nusselt number is in the range $E_{N u^{*}} \in[1.3,18.3] \%$ with a average deviation of $7.5 \%$, whereas that of the friction factor is in the range $E_{f^{*}} \in[3.9,13.8] \%$, with an average difference of $7.4 \%$. The maximum percentage differences noted above are within the same range as those reported in [4] and [23].

The aforementioned agreement in both benchmark tests is indicative of the robustness of the present numerical methodology and the reliability of the corresponding mathematical model.

\section{RESULTS AND DISCUSSION}

The parametric analysis focuses on the effects of Reynolds number $R e$, fin spacing $\delta$, tube diameter $D$ and tube configuration. Although Motamedi et al. [15] used a definition of $R e$ based on diameter $-R e_{D}=u_{i n} D / \nu$ - to study the effects of $R e$ and temperature differences between the fluid at the inlet of the computational domain and the walls, $\Delta T=T_{s}-T_{\text {in }}$ with 
$T_{s}>T_{i n}$, in a fifteen-tube compact heat exchanger, due to the fact that here tube diameter and fin spacing are varied, in addition to $R e_{D}$ we also use a Reynolds number based on fin spacing $R e_{\delta}=u_{i n} \delta / \nu$, as suggested by [16]. However, only the largest value studied by [15]; i.e., $\Delta T=20$, $\mathrm{K}$ with $T_{s}=313 \mathrm{~K}\left(40^{\circ} \mathrm{C}\right)$ and $T_{i n} \equiv \bar{T}_{i n}=293 \mathrm{~K}\left(20^{\circ} \mathrm{C}\right)$ is considered in this study. The corresponding ranges for the Reynolds numbers are: $\operatorname{Re}_{D} \in[120,960]$ and $R e_{\delta} \in[16.5,132]$, for $D=D_{b}$ and $\delta=\delta_{b}$, with $\operatorname{Re}_{\delta}=(\delta / D) R e_{D}$ providing the equivalence between the two definitions. The analysis is simplified by using the baseline values of tube diameter and fin spacing, so that $D=\{0.25,0.5,0.75,1\} D_{b}$ and $\delta=\{0.5,1\} \delta_{b}$, respectively.

\subsection{Methodology of Analysis}

Importantly, in this work we propose the use of the heat rate, instead of averaged $N u$ numbers, since this approach provides higher accuracy [11]-[14], although it may come at the expense of generality. Thus, following the work of [15], results for pressure, streamwise flow velocity and temperature presented here are based on average values given as

$$
\begin{aligned}
\bar{p} & =\frac{1}{A} \int_{A} p d A, \\
\bar{u} & =\frac{1}{A} \int_{A}(\mathbf{u} \cdot \mathbf{n}) d A \\
\bar{T} & =\frac{\int_{A}(\mathbf{u} T \cdot \mathbf{n}) d A}{\int_{A}(\mathbf{u} \cdot \mathbf{n}) d A}
\end{aligned}
$$

where $A$ is the local cross-sectional area of the channel; i.e., $d A=d y d z$, orthogonal to the streamwise direction of the flow at any point along $x, \mathbf{n}$ is the unit vector associated with the surface of interest, and $\mathbf{u}=(u, v, w), T$ and $p$, are the local values of the velocity vector, temperature and pressure.

Energy balances at each section, and between inlet and outlet of the heat exchanger, provide, respectively, the so-called partial, $Q_{j}$, and total, $Q_{T}$, heat rates, which can be quantified by

$$
Q_{j}=\rho \bar{u} A c_{p}\left[\bar{T}\left(x_{j-1}\right)-\bar{T}\left(x_{j}\right)\right],
$$

where $x_{j-1}=x((j-1) \cdot \Delta L)$ and $x_{j}=x(j \cdot \Delta L)$, for $j=1, \ldots, N_{T}$, represent the location of the inlet and outlet planes of the $j$-th section, and

$$
Q_{T}=\sum_{j=1}^{N_{T}} Q_{j}+Q_{L_{x 1}}=\rho \bar{u} A c_{p}\left(\bar{T}_{\text {out }}-\bar{T}_{\text {in }}\right),
$$

where $\bar{T}_{\text {in }}$ and $\bar{T}_{\text {out }}$ are the average temperatures at the inlet and outlet planes of the entire computational domain for the device, and $N_{T}$ is the number of tube sections $\left(N_{T}=6\right)$. The 
term $\Delta L$ above is the length of a specific section of the device that may be selected by the thermal engineer. It is important to note that $Q_{L_{x 1}}$, in Eq. (19), is identified here for the first time as the energy transferred by the combined effect of advection and diffusion to/from the fluid that occurs as the fluid approaches the leading edge of the heat exchanger, and before the fluid interacts with the actual surfaces, due to temperature gradients along the streamwise direction. The effect of the parameters on $Q_{L_{x 1}}$ will be discussed in the next sections.

\subsection{Hydrodynamics and Heat Transfer}

Typical velocity and temperature fields are represented qualitatively by streamlines, isotherms and temperature contours at the mid-plane $z=\delta / 2$, for $\operatorname{Re}_{D}=480, D=D_{b}$ and $\delta=\delta_{b}$, in Figure 6 for the in-line configuration, and in Figure 7 for the staggered arrangement. From the figures it can be seen that, as expected for this value of $R e_{D}$, the flow is nearly uniform at the inlet, develops throughout the heat exchanger length into periodic patterns with recirculation regions behind the tubes, the periodicity being broken at the outlet of the device. A main difference between the in-line and staggered flow-structures is that the latter alternate due to the tube arrangement affecting locally the heat transfer process. This is apparent in both the isotherms and the temperature contours which show that thermal energy is advected downstream in a slightly different manner. On the other hand, a fact more clearly distinguished from the temperature contours is that for both configurations, virtually the total amount of energy transfer has taken place just before the flow reaches the inlet of the fourth tube-section.

Fluid flow patterns and heat transfer behavior similar to the previous case were also noticed for the value of $R e_{D}=960$. At smaller fluid velocities (e.g., $R e_{D}=120$ and $R e_{D}=240$ ), however, though periodic the flow does not display any recirculation, and essentially the entire heat transfer has been achieved at the outlet of the second tube-section. Qualitatively, these results are in agreement to those of [2] for the staggered- and [16] for the in-line-configuration.

It should be noted that at high $R e$ numbers - e.g., $R e_{D}=960-$ unsteady flow patterns are expected. Thus, to ensure that our steady-state model provides accurate solutions, we have also carried out time-dependent analyses - not presented here for brevity- for all the $R e_{D^{-}}$ numbers considered. The corresponding results indicate that with exception of $R e_{D}=960$, for all other values the flow is indeed steady. However, even in the case of $R e_{D}=960$ unsteady flow appears only at the trailing edge of the heat exchanger and its effect on the 
velocity and temperature fields in the device is imperceptible.

\subsection{Reynolds Number Effect}

The effect of Reynolds number in the range $R e_{D}=[120,960]$, for a device with $D=$ $D_{b}$ and $\delta=\delta_{b}$, is analyzed first; the same general trend in the results being found for all the other values of $D$ and $\delta$ considered. Figure 8 displays the cross-section-averaged temperature at several streamwise locations along the heat exchanger for both in-line and staggered configurations, and $R e_{D}=\{120,240,480,960\}$. From the figure it can be seen that regardless of the tube arrangement, as the air travels through the heat exchanger, its temperature increases until reaching the value of the surface temperature of the fins and tubes, $T_{s}$. However, differences are noticeable in the location at which this upper-bound value is attained. For small values of $R e$ (e.g, $R e_{D}=120$ and 240$), T_{s}$ is reached relatively close to the inlet of the device -i.e., within $L / 3$ - whereas for larger inlet velocities (e.g., $R e_{D}=480$ and 960), a longer section of the device -approximately $L / 2$ for $R e_{D}=480$ and nearly the entire length $L$ for $R e_{D}=960$ - is required to achieve it. In addition, the figure also shows that regardless of the local behavior, at low $R e$ numbers the difference between the in-line and staggered mean temperatures is unnoticeable, but as Re increases, such disparity is accentuated in the mid section of the device, the staggered configuration providing higher mean temperatures of the fluid. Importantly, for $R e_{D}=960$ it can be seen that, in the last third section, the temperatures for the in-line device become closer to those of the staggered one until reaching $T_{s}$ at, essentially, the same $x$-location. Using Nusselt numbers, Xie et al. [4] have indicated that the heat transfer is fully developed when the number of tube rows is larger than six. The results here actually show that the streamwise location at which the mean temperature of air reaches that of the wall (at which point no heat transfer occurs) depends on the value of the $R e$ number.

On the other hand, due to advection and diffusion of thermal energy, in both configurations heat transfer to the fluid starts to take place before it actually reaches the leading edge of the heat exchanger. This fact is illustrated in the insert of Figure 8, which shows that the values in the mean temperature of air at the leading edge $(x=0)$, i.e. $\bar{T}(0)$, for $R e_{D}=120$, 240, 480 and 960 , are all $2 \mathrm{~K}$ to $4 \mathrm{~K}$ above the corresponding value at the inlet boundary of the computational domain $\left(T_{i n}=293 \mathrm{~K}\right)$. From the figure it is clear that, as expected, the $R e$ number plays an important role, since the higher values in $\bar{T}(0)$ occur at the smaller $R e$ numbers. As the flow velocity declines from its value at the inlet $u_{i n}$, due to the presence of the fins and tubes, heat transfer by diffusion becomes important, particularly at low 
Re numbers in which the residence time is larger. As Re increases and the residence time decreases, the values of $\bar{T}(0)$ become closer to that of $T_{i n}$. Finally, the tube arrangement also influences the values of $\bar{T}(0)$, being these slightly higher for in-line than for staggered, since the latter generates greater resistance to the airflow.

\subsection{Tube Diameter Effect}

The attention is now shifted to examine the effect of varying the tube diameter on the fluid flow and energy transfer for the two configurations. Four values, that are defined as fractions of the baseline diameter, i.e., $D=\{0.25,0.5,0.75,1\} D_{b}$, along with the a set values for $R e_{\delta}=\{16.5,22,66,132\}$ which are equivalent to those used in the previous section, and $\delta=\delta_{b}$, are considered here. The heat transfer is presented in terms of the ratio $Q_{j} / Q_{T}$; i.e., the fraction of the total heat rate that is transferred at each section of the specific device; the pressure drop $\Delta \bar{p}$ is used for the hydrodynamics. Figure 9 depicts the streamwise distribution of the cross-section-averaged pressure along the over-tube channel of the heat exchanger. The corresponding results for $R e_{\delta}=66$ and 132 are shown as a reference, the same general trend being found for $R e_{\delta}=16.5$ and 33. From the figure it can be observed that, as expected, the pressure decreases linearly along the channel and a higher value of $R e$ (larger inlet velocity) generates a higher pressure drop. On the other hand, by focusing on the effect of tube-diameter variation it is clear that for a given $R e_{\delta}$, in both configurations, as the diameter decreases so does the pressure drop since smaller tube-diameters reduce blockage of the flow. In agreement with other studies in the literature, the staggered arrangement gives rise to larger pressure drop; however, for a fixed Re number, larger values of $D$ induce a bigger disparity in $\Delta \bar{p}$ between the in-line and staggered layouts. These differences in the values of $\Delta \bar{p}$ between the two configurations also increase with $R e_{\delta}$, being negligible for $R e_{\delta}=16.5$ and 33, and significant for $R e_{\delta}=132$; although it is apparent that, for the case under analysis, variations in the $R e$ number have a smaller impact than those of tube-diameter.

The results for $Q_{j} / Q_{T}$ for the two tube-configurations of this study are presented in Figures 10, 11 and 12, for $R e_{\delta}=33,66$ and 132, respectively; the value of $R e_{\delta}=16.5$ yields a similar but less interesting outcome. From the figures it can be seen that regardless of the Re number, and tube arrangement, the fraction of heat rate transferred at each tube-fin section, $Q_{j} / Q_{T}$, attains its largest value in the first section and declines towards zero in an exponential-like fashion as the fluid passes by the other sections that encompass the over-tube channel of the corresponding heat exchanger. However, details of the $Q_{j} / Q_{T}$-distribution, 
and hence the actual values at each fin-tube section, strongly depend on the $R e$ number, and the diameter $D$. For $R e_{\delta}=33$, for example, approximately $86 \%$ of the total heat transferred from the walls to the fluid occurs in section 1 , only $2 \%$ of it takes place in section 2 and there is essentially no transfer of energy downstream of it. In comparison, as $R e_{\delta}$ increases, the value of $Q_{j} / Q_{T}$ in the first section decreases, while rising at the other sections. For $R e_{\delta}=66$ the corresponding fraction in section 1 lies in the range $76.8-83.3 \%$, whereas its values are within $7.4-12.3 \%$ and $0.7-2.8 \%$ in sections 2 and 3 , respectively, beyond which the amount of energy exchanged is negligible. For $R e_{\delta}=132$, the ranges for $Q_{j} / Q_{T}$ are: $59.5 \%-67.7 \%$ in section $1,17.6 \%-20 \%$ in section $2,5.2 \%-8.6 \%$ and $1.6 \%-4.1 \%$ in sections 3 and 4 , respectively, and less than $2 \%$ in section 5 . These results are expected since for low $R e$ numbers (e.g., $R e_{\delta}=33$ ) the residence time of air at each section is larger than that for higher $R e_{\delta}$ (e.g., $R e_{\delta}=33$ and 132). Longer residence times produce bigger changes in temperature of the fluid, hence greater values of heat transfer, especially in the sections closer to the inlet where the temperature difference between the walls and the fluid is larger, as observed earlier in Figure 8.

In addition to the fraction of the total heat rate that is transferred at each section of the heat exchanger, Figures 10-12 illustrate the fraction of the total heat transfer rate that takes place before the air reaches the leading edge of the device. From the figures it can be seen that, again, regardless of the tube configuration, as $R e_{\delta}$ increases from 33 to 132 , the fraction $Q_{L_{x 1}} / Q_{T}$ decreases from $11.8 \%$ for $R e_{\delta}=33$ to $6.7 \%$ when $R e_{\delta}=132$, with a value of $8 \%$ for $R e_{\delta}=66$. Once more, these results are in agreement with those of the previous section, where it was shown that the mean temperature of air at the leading edge $\bar{T}(0)$, declines with increasing Re number (see, Figure 8). When the contributions from $Q_{j} / Q_{T}$ and $Q_{L_{x 1}} / Q_{T}$ are taken into account [c.f. Eq. (19)], it can be seen that, regardless of the value of tube diameter $D$ and tube configuration, for a given device operating at $R e_{\delta}=33$ only the first section (i.e., $L / 6$ ) is needed to transfer $99 \%$ of the total heat rate that could be exchanged. For a device operating at $R e_{\delta}=66$, three sections (i.e., $L / 2$ ) are necessary to transfer $99 \%$ of the heat rate that could be potentially achieved, whereas for $R e_{\delta}=132$, the number of required sections for the given device is five (equivalently to a length $5 L / 6$ ). It is important to note, however, that the total energy actually transferred in Watts [W] by a given device that operates at higher Re-number flowrate is larger than that operating with smaller flowrates (i.e., smaller Re number).

On the other hand, is should be pointed out that variations in the magnitude of $Q_{j} / Q_{T}$ at each tube-fin section indicated above are due to differences in the size of tube diameter. From 
the set of figures 10-12, it can be seen that the way tube diameter influences the magnitude of the fraction of the total heat transfer rate at each tube-fin section is closely related to the Reynolds number. For small values of $\operatorname{Re}_{\delta}\left(R e_{\delta}=16.5\right.$ and 33$)$ variations in tube diameter do not have a significant effect on the fraction of heat transfer at each section; e.g., differences in its value at section 1 are confined to less than $2 \%$ when the diameter changes in the range $\left[0.25 D_{b}, D_{b}\right]$. However, at larger Re-numbers the tube diameter progressively impacts the value of $Q_{j} / Q_{T}$ at each section. For $R e_{\delta}=66$, e.g., a device with an in-line tube configuration and diameter $D=D_{b}$, has a value for $Q_{j} / Q_{T}$ in tube section 1 of $76.8 \%$, but if the diameter is reduced to $D=0.25 D_{b}$, the fraction of the total heat rate increases by $8.5 \%$ (the comparable increment in a device with a staggered configuration is $7.6 \%$ ). These are foreseen results since, in general, smaller tube diameters provide greater exposure of the fluid to the fin surface. For the same value of $R e_{\delta}$, a decrease in diameter from $D_{b}$ to $0.25 D_{b}$, causes the contrary effect in sections 2 and 3 ; i.e., a decline of $38.1 \%$ and $73.2 \%$ (39.3\% and $64.6 \%$ for the staggered case) in the fraction $Q_{j} / Q_{T}$, respectively, where the larger percentages are due to smaller reference values. This behavior is a consequence of the heat transfer process carried out in section 1, which promotes -for smaller diametersthe air temperature to raise its value closer to that of the fins and tubes, thus reducing the temperature difference between fluid and walls in the remaining sections. For $R e_{\delta}=132$, the results are analogous but more noticeable than those of the previous case, where a reduction in the diameter from $D_{b}$ to $0.25 D_{b}$ causes an increase in the fraction of the total heat rate from its baseline values of $13.8 \%$ and $12.4 \%$ in section 1 for the in-line and staggered configurations, respectively. In sections 2,3 and 4 , the corresponding decline in the values $Q_{j} / Q_{T}$ are, respectively: $2.3 \%, 38.3 \%$ and $60.8 \%$ for the in-line alignment and $11 \%, 32.7 \%$ and $56.8 \%$ for the staggered configuration. Therefore, as flow velocity increases, the effects of tube diameter on heat transfer process are amplified. Finally, as seen from the results, both the fraction of the total heat transfer and the temperature distributions exhibit similar trends in the in-line- and staggered-tube configurations, and although distinct in the details, in average there are no significant differences in the fraction of heat transfer rate, and its streamwise distribution, between the two configurations.

\subsection{Fin Spacing Effect}

The influence of fin spacing on the heat transfer rate is assessed by considering two values: the baseline fin spacing $\delta=\delta_{b}=4.4 \mathrm{~mm}$, and a value half of it $\left(\delta=\delta_{b} / 2=2.2 \mathrm{~mm}\right)$. Since all the results in the previous section were obtained with the value of $\delta=\delta_{b}$, for Reynolds 
numbers $R e_{\delta}=33,66$ and 132, in the present case we only focus on $R e_{\delta}=132$, equivalent to $R e_{D}=960$ with $D=D_{b}$, which is the most relevant case. In all the numerical tests, the corresponding inlet velocity, $u_{i n}$, is maintained at $0.46 \mathrm{~m} / \mathrm{s}$. Figures 12 and 13 show, respectively, the fraction of the total energy $\left(Q_{j} / Q_{T}\right)$ that is transferred at each section of the device with $\delta=\delta_{b}$ and $\delta=\delta_{b} / 2$, for the two tube configurations. From the figures it can be seen that for the given $R e_{D}$ number, and independently of the tube arrangement, the results for the two values of fin spacing illustrate a different behavior in both the $Q_{j} / Q_{T^{-}}$ distribution and its actual values. For the case of $\delta=\delta_{b} / 2$ the values of $Q_{j} / Q_{T}$ are higher at section 1 (e.g., $86.6 \%$ for in-line- and $86.5 \%$ for staggered-layout), and smaller at the other sections (2.6\% and $2.5 \%$ in section 2 , respectively, for the in-line and staggered alignments), than those previously observed for the case of $\delta=\delta_{b}$. In addition, although the influence of the diameter on the fraction of the total heat rate is relevant for the device with a fin spacing $\delta_{b}$, its impact for the device with a fin spacing $\delta / 2$ is negligible.

By taking a look at Figure 10, it can be observed that the aforementioned results resemble those of $R e_{D}=240$ (with $\delta=\delta_{b}$ ), where, $Q_{j} / Q_{T}$ in section 1 is approximately $86 \%$ and around $2 \%$ in section 2 , being negligible in the subsequent sections. The values of $Q_{L_{x 1}} / Q_{T}$ for $R e_{D}=960$ and $\delta_{b} / 2$ and those of $R e_{D}=240$ and $\delta_{b}$ are also extremely close. Therefore, it is apparent that the effect of reducing the fin spacing is essentially the same as that of reducing the value of $R e_{D}$ for a fixed $\delta$. It is to be noted that devices with larger fin spacings (an optimum value for a single tube-row configuration has been reported by Romero-Mendez et al. [16]) require a larger number of sections for the fluid to attain the value of the surface temperature due to greater quantity of fluid at each section. From Figures 12 and 13, e.g., a device with $\delta=\delta_{b}$, requires at least five tubes (a length $5 L / 6$ ) to achieve $99 \%$ of the energy transfer that could potentially be transferred. By reducing the fin spacing to $\delta=\delta / 2$, after two sections the device can no longer transfer energy to the fluid; i.e., the total amount that could be potentially achieved is reached at a streamwise position $L / 3$.

\section{CONCLUDING REMARKS}

Plate-fin and tube compact heat exchangers are widely used in industrial applications, and because of their importance, designing efficient systems is essential. Thermo-physical properties of the over-tube fluid and the often laminar nature of the flow in this type of equipment, however, generate a thermal resistance that critically constrains the transfer of energy. Therefore, it is crucial to ensure efficient heat transfer in order to minimize environ-

mental impact while maximizing device compactness so that operating and manufacturing 
costs can also be reduced. These objectives can be accomplished by means of numerical solutions of mathematical models, which have proven to be effective for the analysis of thermal devices. Despite the fact that experiments are useful to study specific scenarios, numerical simulations enable more comprehensive investigations.

In this study, the hydrodynamic and heat transfer characteristics of compact fin and tube heat exchangers have been investigated numerically by introducing a methodology of analysis based on local and global energy balances, from three-dimensional velocity and temperature fields, to design more efficient devices. The approach is useful in providing, at the expense of some generality, high accuracy and clear information to better understand the convection heat transfer process in compact heat exchangers, since intermediate steps via Nusselt numbers and heat transfer coefficients are not necessary.

Using the procedure, along with the concept of fraction of the total heat rate available for the specific device, the results have shown that, overall, the Reynolds number Re (i.e., fluid velocity), plays an important role, whereas the role of tube layout is minor, the effects of tube diameter and fin spacing being closely related to the magnitude of the Re number. It has been found that the fraction of the total heat rate has an exponential-like-decay streamwise distribution, the actual shape of which strongly depends on Reynolds number, diameter size and fin spacing. For small values of $R e, 99 \%$ of the heat rate that could be potentially achieved occurs closer to the inlet of the device, whereas for large Re numbers, nearly the entire length is necessary. On the other hand, the influence of the tube-diameter on both heat transfer and pressure drop is negligible for small values of Reynolds number but progressively impacts both quantities as $R e$ increases. For a given $R e$ number, however, as tube-diameter is reduced so does the pressure drop. A similar -but less pronouncedeffect to that of Re number on the heat rate is produced by fin spacing, where the influence of tube-diameter is relevant only for larger values of the fin spacing. From the results it is apparent that the effect of reducing the fin spacing is relatively similar to that of reducing the Reynolds number. In addition, although different in the details, it has been found that both in-line and staggered configurations provide similar values of the heat transfer; however, differences in pressure drop between the two layouts exist and become more pronounced as Re number increases, the values of the staggered alignment being higher. Finally, changes in fluid temperature -and hence energy transfer to/from it- that occur even before the fluid interacts with the actual surfaces, and their dependence on the Reynolds number, have been identified and quantified for the first time. Larger Re numbers provide smaller values of the relative energy transferred before the fluid reaches the device. However, the effects of tube 
diameter and tube alignment are negligible.

The results from this work suggest that, when designing more efficient compact heat exchangers, parameters such as fin spacing, tube diameter and alignment, and flow velocity should be analyzed simultaneously. Using the methodology proposed in this study, further investigation will focus on the interactions of these and other geometrical parameters in the operation of compact heat exchangers and their subsequent optimization.

\section{ACKNOWLEDGMENTS}

Jeanette Cobian-Iñiguez and Angela Wu have been recipients, respectively, of an LSAMPBD fellowship (NSF HRD-1246662) and a CEaS-CSULA fellowship (NSF HRD-0932421), for which we are grateful. Florian Dugast is thankful for the financial support received from CEaS-CSULA for a summer internship. This project has been partially supported by NSF grants HRD-0932421 and ARA-R2-0963539.

\section{References}

[1] M. Fiebig, A. Grosse-Gorgemann, Y. Chen, and N.K. Mitra. Conjugate heat transfer of a finned tube Part A: heat transfer behavior and occurrence of heat transfer reversal. Numerical Heat Transfer A, 28:133-146, 1995.

[2] J.-Y. Jang, M.-C. Wu, and W.-J. Chang. Numerical and experimental studies of three dimensional plate-fin and tube heat exchangers. Int. J. Heat and Mass Transfer, 39(14):3057-3066, 1996.

[3] Y.L. He, W.Q. Tao, F.Q. Song, and W. Zhang. Three-dimensional numerical study of heat transfer characteristics of plain plate fin-and-tube heat exchangers from view point of field synergy principle. International Journal of Heat and Fluid Flow, 26:459-473, 2005.

[4] G.N. Xie, Q.W. Wang, and B. Sunden. Parametric study and multiple correlations on air-side heat transfer and friction characteristics of fin-and-tube heat exchangers with large number of large-diameter tube rows. Applied Thermal Engineering, 29:1-16, 2009.

[5] A. Erek, B. Ozerdem, L. Bilir, and Z. Ilken. Effect of geometrical parameters on heat transfer and pressure drop characteristics of plate fin and tube heat exchangers. Applied Thermal Engineering, 25:2421-2431, 2005.

[6] M. Abu Madi, R.A. Johns, and M.R. Heikal. Performance characteristics correlation for round tube and plate finned heat exchangers. Int. J. Refrigeration., 21(7):507-517, 1998.

[7] S.M. Saboya and F.E.M. Saboya. Experiments on elliptic sections in one- and two-row arrangements of plate fin and tube heat exchangers. Experimental Thermal and Fluid Science, 24:67-75, 2001. 
[8] S.F. Tsai and T.W.H. Sheu. Some physical insights into a two-row finned-tube heat transfer. Computers \& Fluids, 27(1):29-46, 1998.

[9] M. Tutar and A. Akkoca. Numerical analysis of fluid flow and heat transfer characteristics in three-dimensional plate fin-and-tube heat exchangers. Numer. Heat Transfer, Part A, 46:301-321, 2004.

[10] L.-Z. Zhang, W.-C. Zhong, J.-M. Chen, and J.-R. Zhou. Fluid flow and heat transfer in plate-fin and tube heat exchangers in a transitional flow regime. Numer. Heat Transfer, Part A, 60:766-784, 2011.

[11] A. Pacheco-Vega, M. Sen, K.T. Yang, and R.L. McClain. Genetic-algorithm-based predictions of fin-tube heat exchanger performance. In J.S. Lee, editor, Proceedings of the Eleventh International Heat Transfer Conference, volume 6, pages 137-142, New York, NY, August 1998. Taylor \& Francis.

[12] A. Pacheco-Vega, G. Díaz, M. Sen, K.T. Yang, and R.L. McClain. Heat rate predictions in humid air-water heat exchangers using correlations and neural networks. ASME J. Heat Transfer, 123(2):348-354, 2001.

[13] A. Pacheco-Vega, M. Sen, K.T. Yang, and R.L. McClain. Neural network analysis of fin-tube refrigerating heat exchanger with limited experimental data. Int. J. Heat and Mass Transfer, 44(4):763-770, 2001.

[14] A. Pacheco-Vega, M. Sen, and K.T. Yang. Simultaneous determination of in- and overtube heat transfer correlations in heat exchangers by global regression. Int. J. Heat and Mass Transfer, 46(6):1029-1040, 2003.

[15] A. Motamedi, A. Pacheco-Vega, and J.R. Pacheco. Numerical analysis of a multi-row multi-column compact heat exchanger. Journal of Physics: Conference Series, 395:1-9, Paper 012047, 2012.

[16] R. Romero-Mendez, M. Sen, K.T. Yang, and R. McClain. Effect of fin spacing on convection in a plate fin and tube heat exchanger. Int. J. Heat and Mass Transfer, 43:39-51, 2000.

[17] R. Romero-Mendez, R. Adame, and M. Sen. Study of the parameters affecting conjugate heat transfer in plate-fin and tube heat exchanger (in Spanish). Interciencia, 26(8):321$326,2001$.

[18] J.R. Pacheco. The solution of viscous incompressible jet flows using non-staggered boundary fitted coordinate methods. Int. J. Numer. Methods Fluids, 35:71-91, 2001.

[19] J.R. Pacheco and A. Pacheco-Vega. Analysis of thin film flows using a flux vector splitting. J. Fluids Engineering., 125:365-374, 2003.

[20] J.R. Pacheco, A. Pacheco-Vega, T. Rodić, and R.E. Peck. Numerical simulations of heat transfer and fluid flow problems using an immersed-boundary finite-volume method on non-staggered grids. Numer. Heat Transfer, Part B, 48:1-24, 2005.

[21] A. Pacheco-Vega, J.R. Pacheco, and T. Rodić. A general scheme for the boundary conditions in convective and diffusive heat transfer with immersed boundary methods. ASME J. Heat Transfer, 129:1506-1516, 2007. 
[22] H.J. Kang, W. Li, H.Z. Xin, and W.Q. Tao. Experimental study on heat transfer and pressure drop characteristics of four types of plate fin-and-tube heat exchanger surfaces. Journal of Thermal Science, 3(1):34-42, 1994.

[23] F. Zhou, N.E. Hansen, D. Geb, and I. Catton. Determination of the number of tube rows to obtain closure for volume averaging theory based model of fin-and-tube heat exchangers. ASME Journal of Heat Transfer, 133(121801), 2011. 


\section{FIGURE CAPTIONS}

1. Schematic of a compact fin-tube heat exchanger. (a) In-line alignment. (b) Staggered alignment.

2. Minimum heat exchanger computational domain for: (a) in-line configuration and (b) staggered configuration.

3. Schematic of the type of mesh used in the computational domain. (a) In-line alignment. (b) Staggered alignment.

4. Grid independence tests for $R e_{D}=480$. ( $\left.-\mathbf{-}-,-\square-\right)$ streamwise velocity $u ;(-\boldsymbol{\Lambda}-,-\triangle-)$ pressure $p$. Solid symbols are for in-line and open symbols are for staggered configurations, respectively.

5. Comparison of results from present model $(\diamond)$ with: (a) numerical simulations $(-)$ of Romero-Mendez et al. [16]; (b) experiments (ם), and correlation $(-\cdot-)$ of Kang et al. $[22]$.

(a) $N u^{*}, N u^{*} / S^{*}$ and $\Delta p^{*}$ vs. $S^{*}$, for $R e^{*}=630$.

(b) $N u^{*}$ and $f^{*}$ vs. $R e^{*}$.

6. Streamlines, isotherms and temperature contours for in-line configuration, $R e_{D}=480$, $D=D_{b}$ and $\delta=\delta_{b}$.

7. Streamlines, isotherms and temperature contours for staggered configuration, $R e_{D}=$ $480, D=D_{b}$ and $\delta=\delta_{b}$.

8. Streamwise distribution of $\bar{T}$ for $D=D_{b}, \delta=\delta_{b}$ and different $R e_{D}$. (- $\left.\bullet-,-\circ-\right)$ $R e_{D}=120 ;(-\boldsymbol{\Delta}-,-\triangle-) R e_{D}=240 ;\left(--_{-},-\diamond-\right) R e_{D}=480 ;(-\mathbf{Q}-,-\square-) R e_{D}=$ 960. Solid symbols are for in-line and open symbols are for staggered configurations, respectively. The symbols showing the in-line and staggered averaged-temperatures at the inlet $(x=0)$ of the heat exchanger have been shifted horizontally for clarity.

9. Streamwise distribution of $\bar{p}$ for $\delta=\delta_{b}, R e_{\delta}=66$ and $R e_{\delta}=132$, and different tube diameters. (-口-, $-\square-) D=0.25 D_{b} ;\left(-\nabla_{-},-\diamond-\right) D=0.5 D_{b} ;(-\boldsymbol{\Delta}-,-\triangle-)$ $D=0.75 D_{b} ;(-\bullet-,-\circ-) D=D_{b}$. Solid symbols are for in-line and open symbols are for staggered configurations, respectively.

10. Fraction of heat rate $Q_{j} / Q_{T}$ for $R e_{\delta}=33, \delta=\delta_{b}$ and different values of $D$. $\left.D=0.25 D_{b} ;(\diamond, \diamond) D=0.5 D_{b} ;(\boldsymbol{\Lambda}, \triangle) D=0.75 D_{b} ; \bullet \bullet, \circ\right) D=D_{b}$.

(a) In-line configuration.

(b) Staggered configuration.

11. Fraction of heat rate $Q_{j} / Q_{T}$ for $R_{\delta}=66, \delta=\delta_{b}$ and different values of $D$. $D=0.25 D_{b} ;(\diamond, \diamond) D=0.5 D_{b} ;(\boldsymbol{\Lambda}, \triangle) D=0.75 D_{b} ;(\bullet, \circ) D=D_{b}$.

(a) In-line configuration.

(b) Staggered configuration. 
12. Fraction of heat rate $Q_{j} / Q_{T}$ for $R e_{\delta}=132, \delta=\delta_{b}$ and different values of $D$. $D=0.25 D_{b} ;(\diamond, \diamond) D=0.5 D_{b} ;(\boldsymbol{\Lambda}, \triangle) D=0.75 D_{b} ;(\bullet, \circ) D=D_{b}$.
(a) In-line configuration.
(b) Staggered configuration.

13. Fraction of heat rate $Q_{j} / Q_{T}$ for $R e_{D}=960, \delta=\delta_{b} / 2$ and different values of $D$. $D=0.25 D_{b} ;(\diamond, \diamond) D=0.5 D_{b} ;(\boldsymbol{\Delta}, \triangle) D=0.75 D_{b} ;(\bullet, \circ) D=D_{b}$.

(a) In-line configuration.

(b) Staggered configuration. 


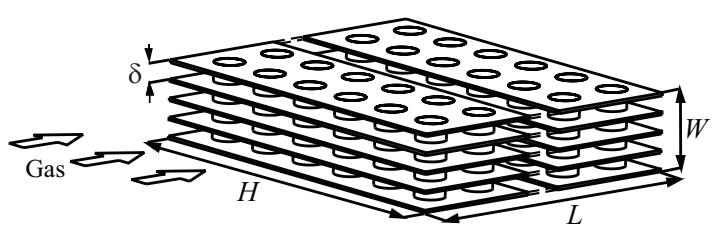

(a)

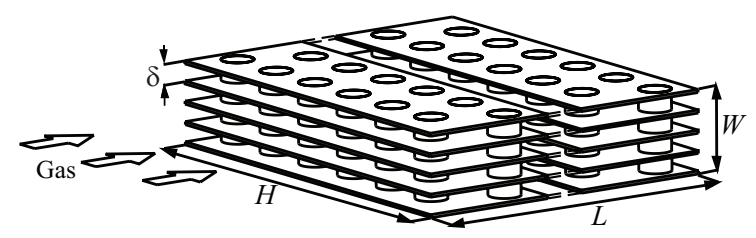

(b)

Figure 1: Schematic of a compact fin-tube heat exchanger. (a) In-line alignment. (b) Staggered alignment. 


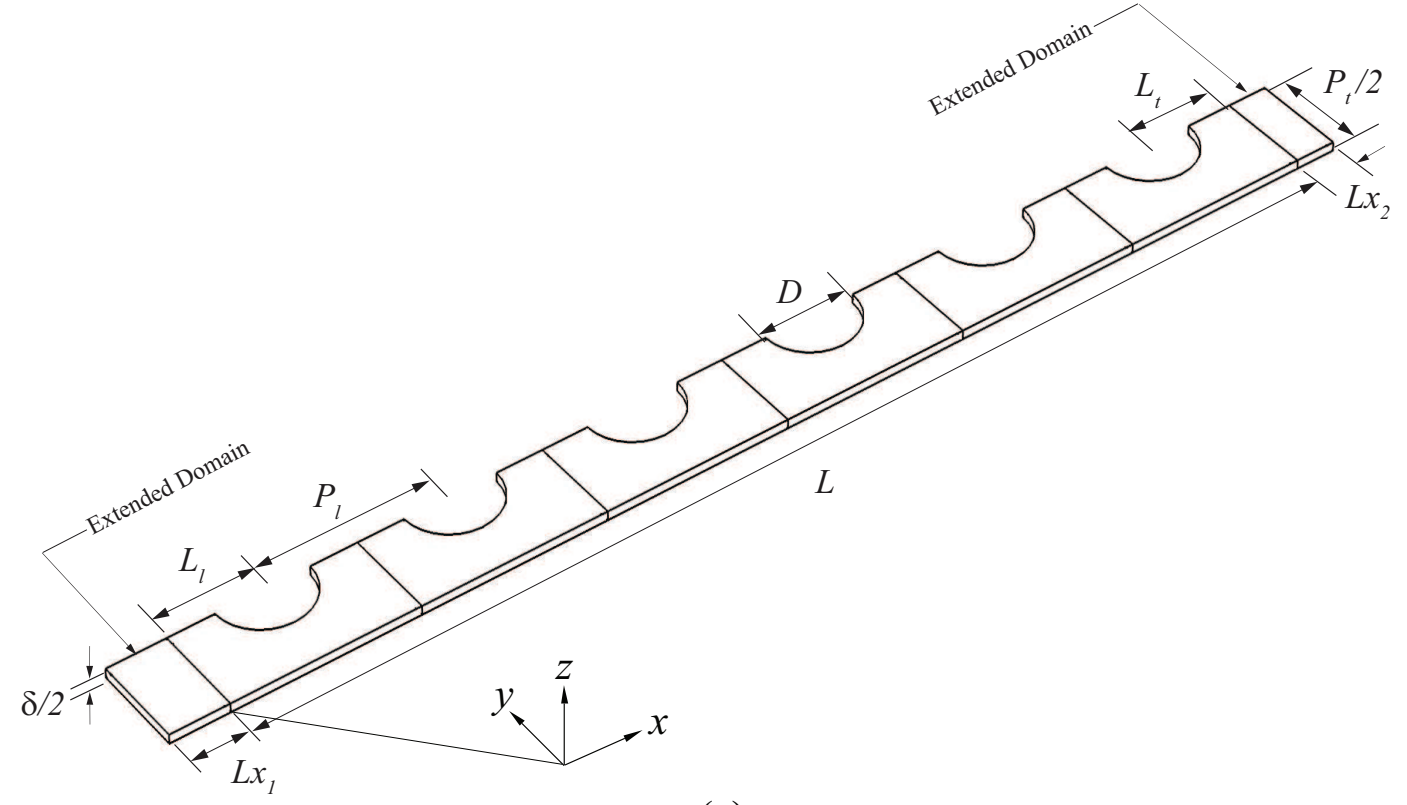

(a)

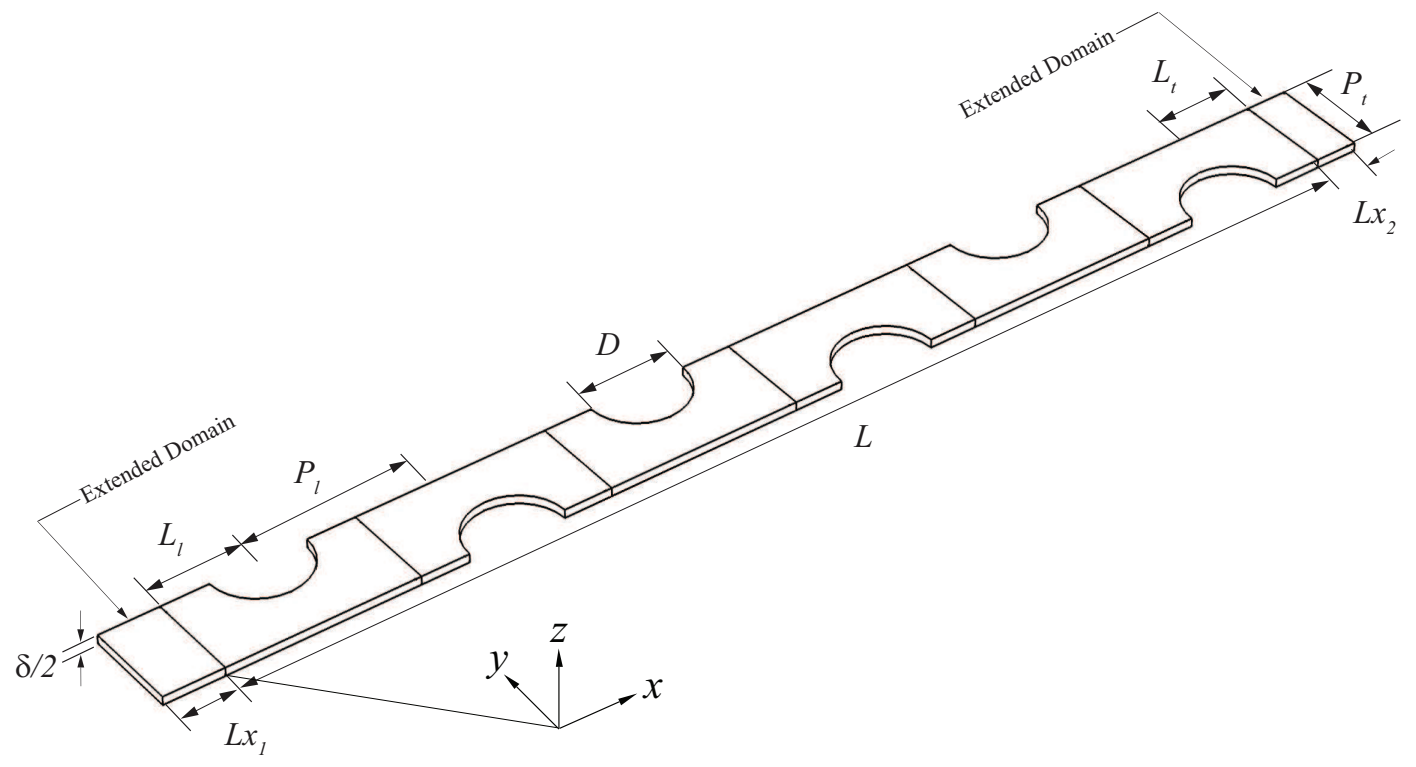

(b)

Figure 2: Minimum heat exchanger computational domain for: (a) in-line configuration and (b) staggered configuration. 25 


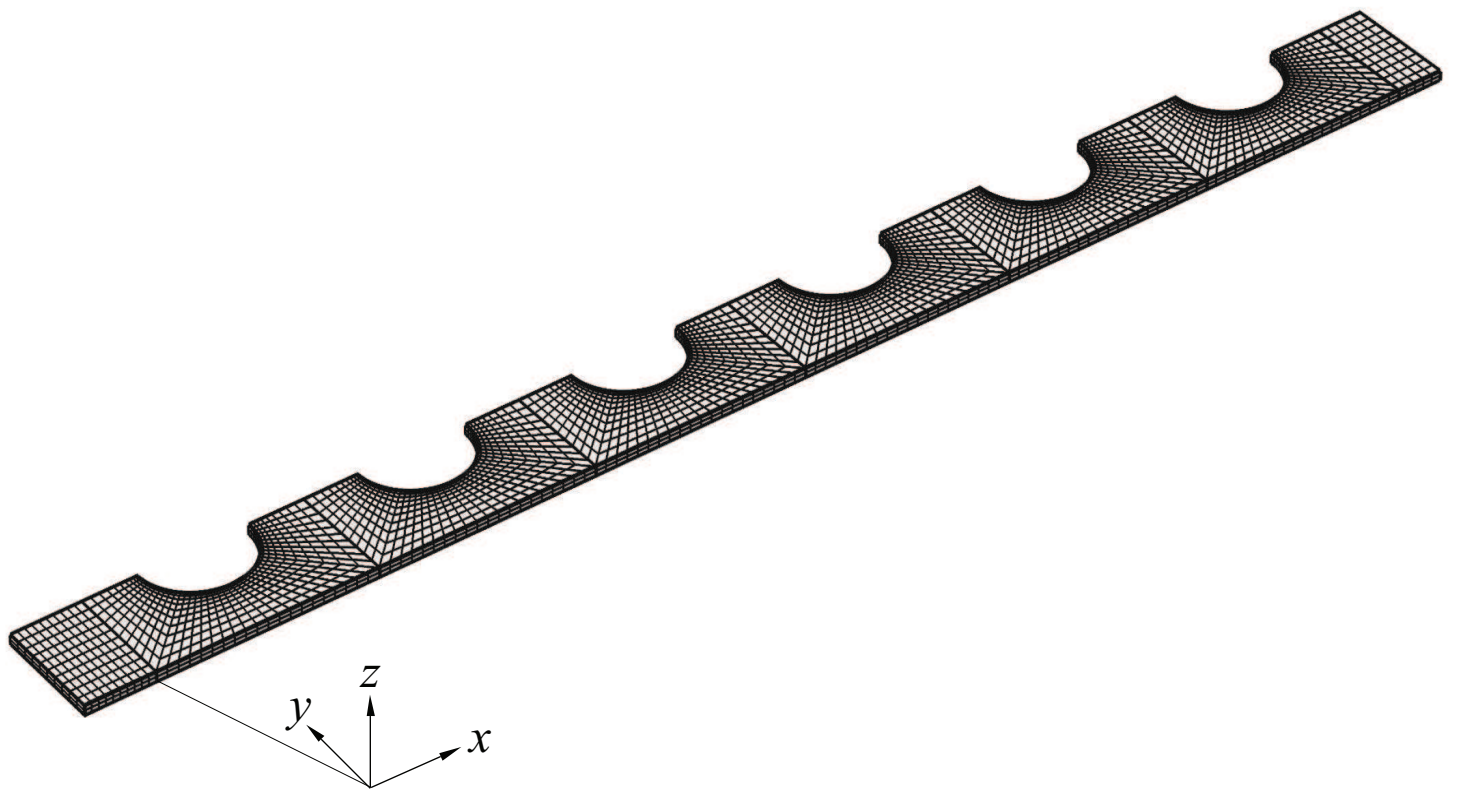

(a)

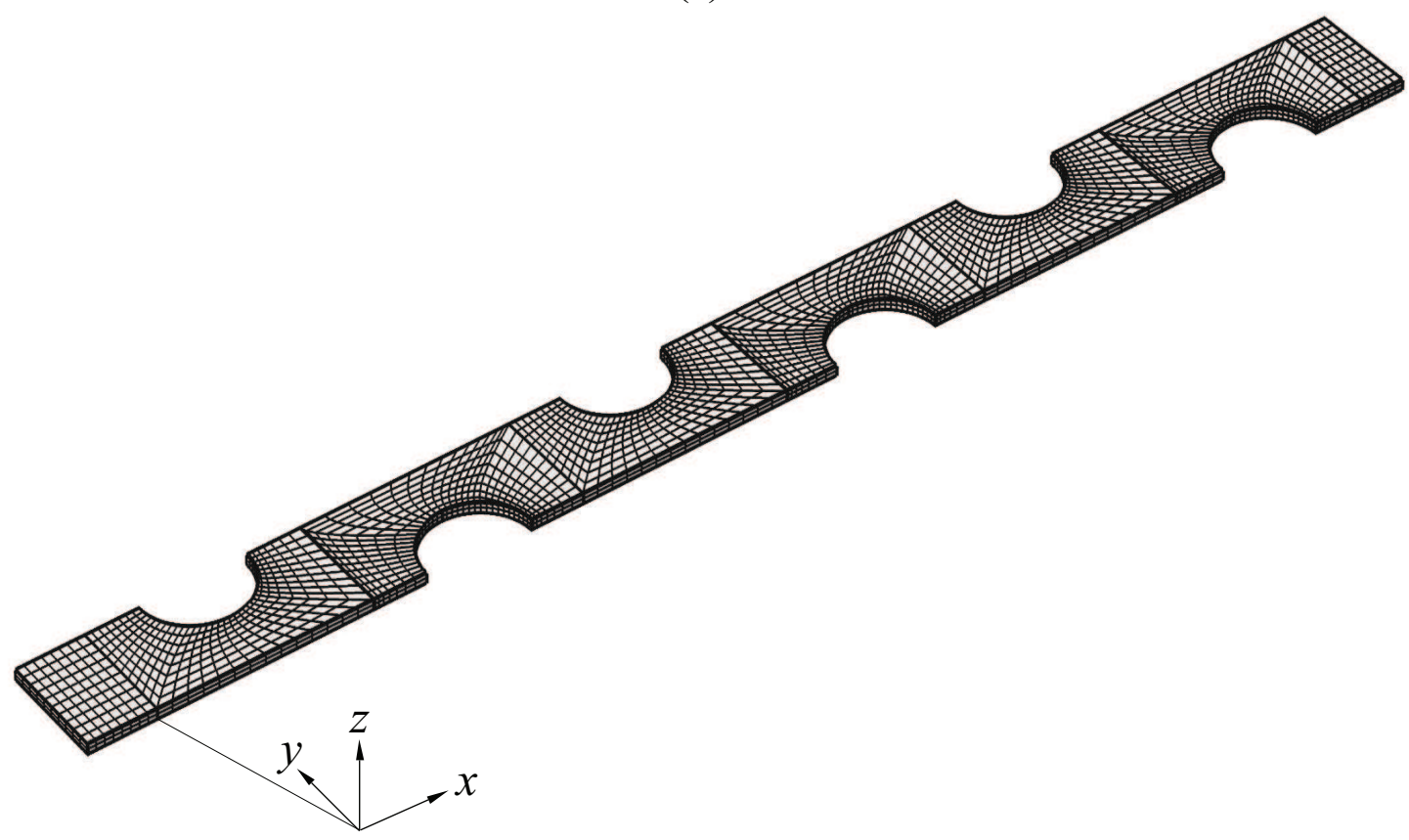

(b)

Figure 3: Schematic of the type of mesh used in the computational domain. (a) In-line alignment. (b) Staggered alignment. 


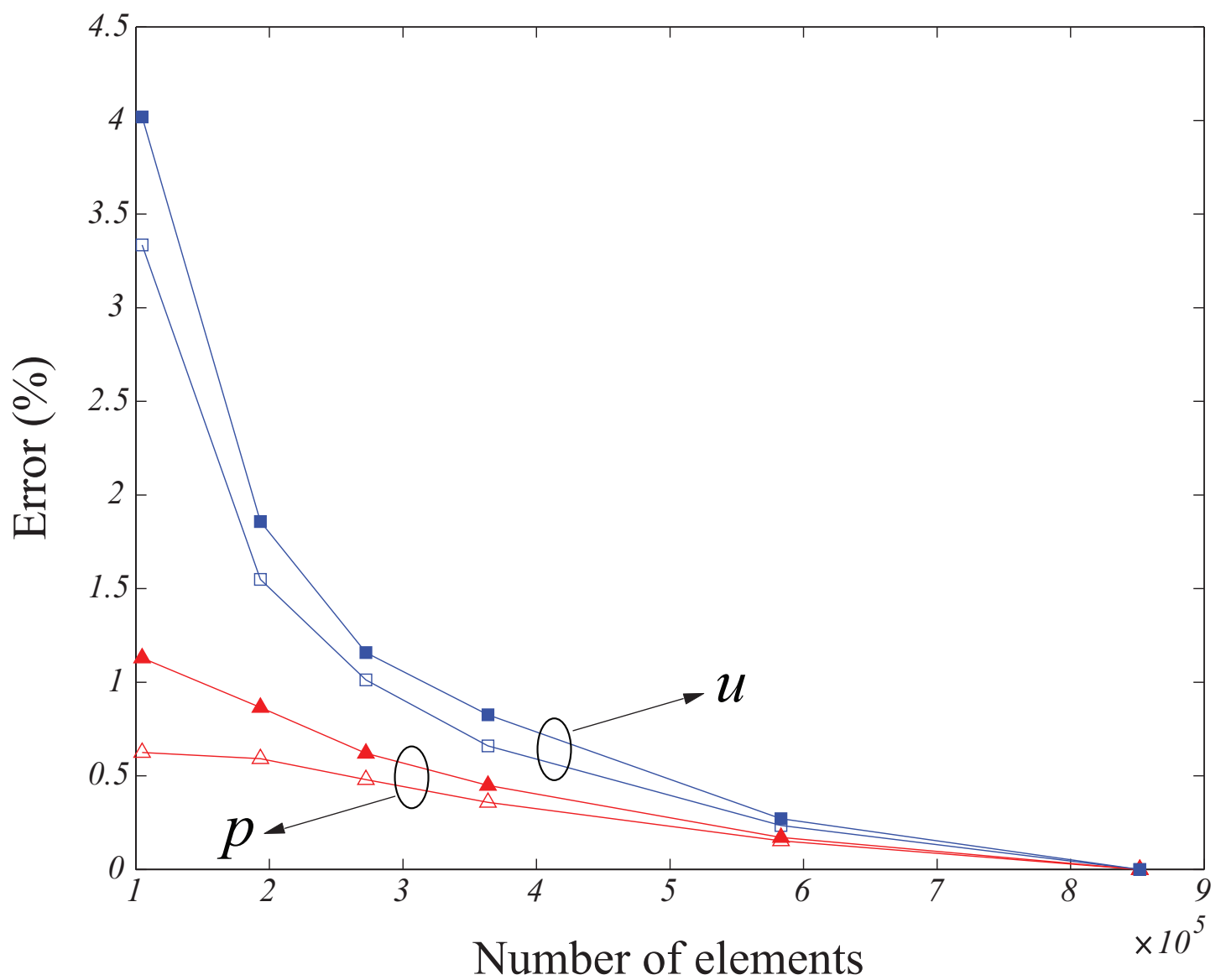

Figure 4: Grid independence tests for $R e_{D}=480$. (-口-, $\left.-\square-\right)$ streamwise velocity $u$; $(-\boldsymbol{\Delta}-,-\triangle-)$ pressure $p$. Solid symbols are for in-line and open symbols are for staggered configurations, respectively. 


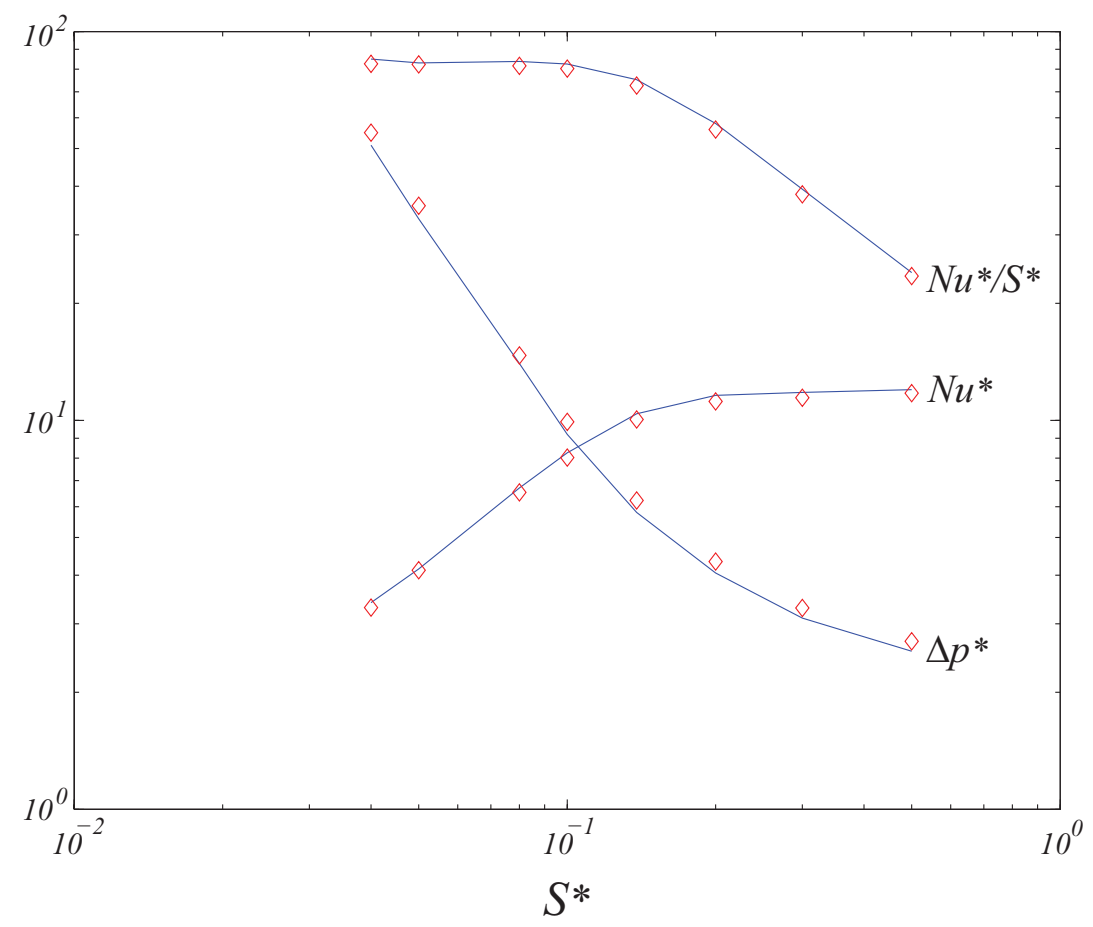

(a) $N u^{*}, N u^{*} / S^{*}$ and $\Delta p^{*}$ vs. $S^{*}$, for $R e^{*}=630$.

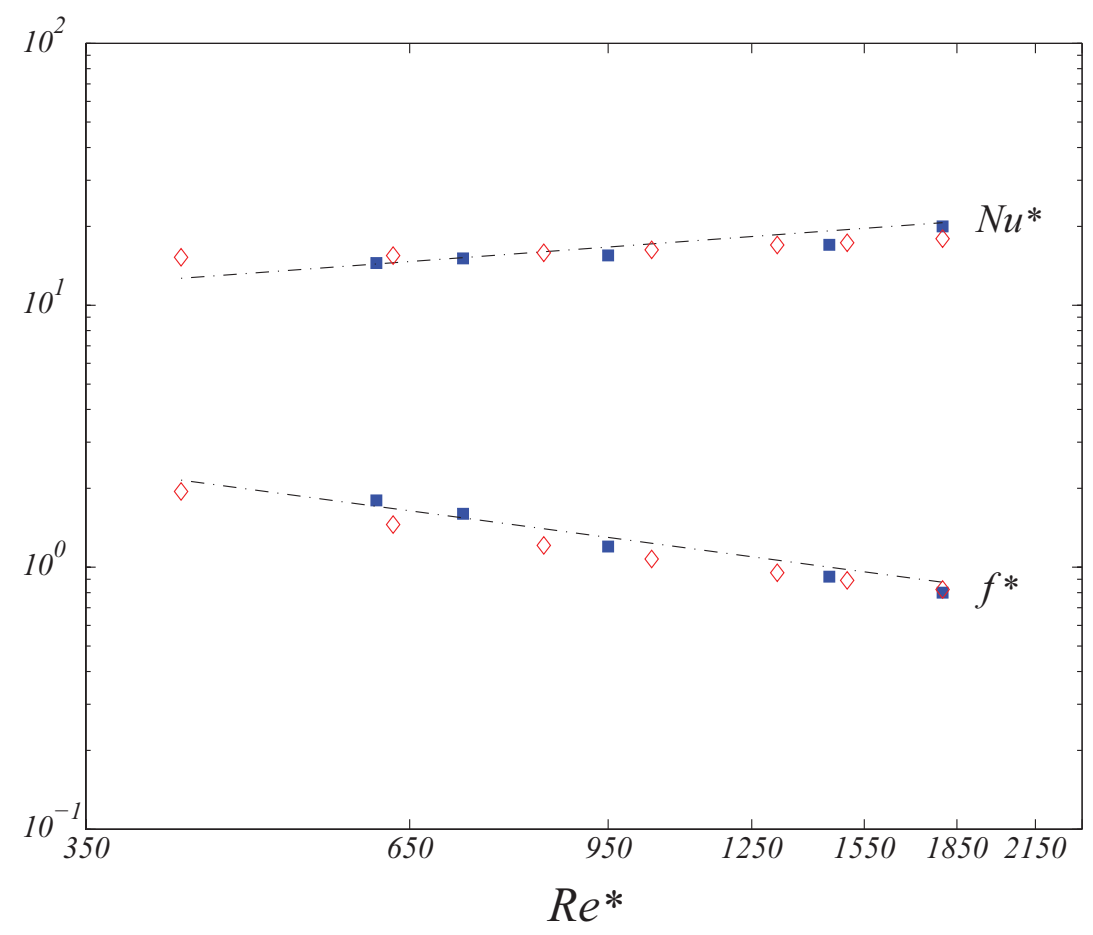

(b) $N u^{*}$ and $f^{*}$ vs. $R e^{*}$.

Figure 5: Comparison of results from present model $(\diamond)$ with: (a) numerical simulations $(-)$ of Romero-Mendez et al. [16]; (b) experiments ( $)$, and correlation (- - - ) of Kang et al. [22]. 


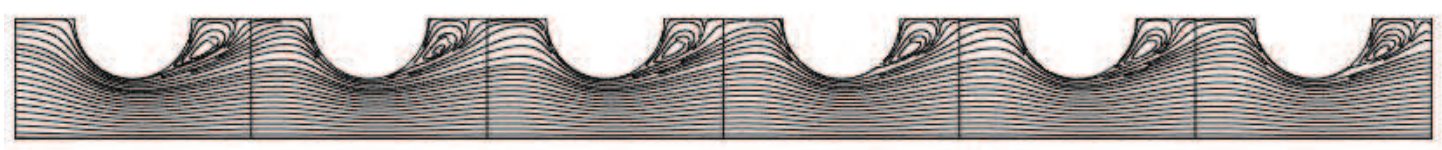

(a) Streamlines.

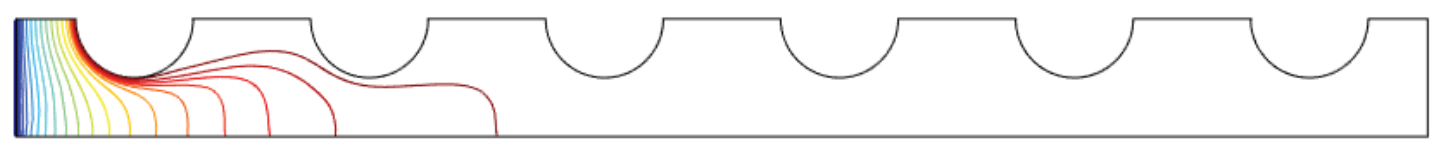

(b) Isotherms.

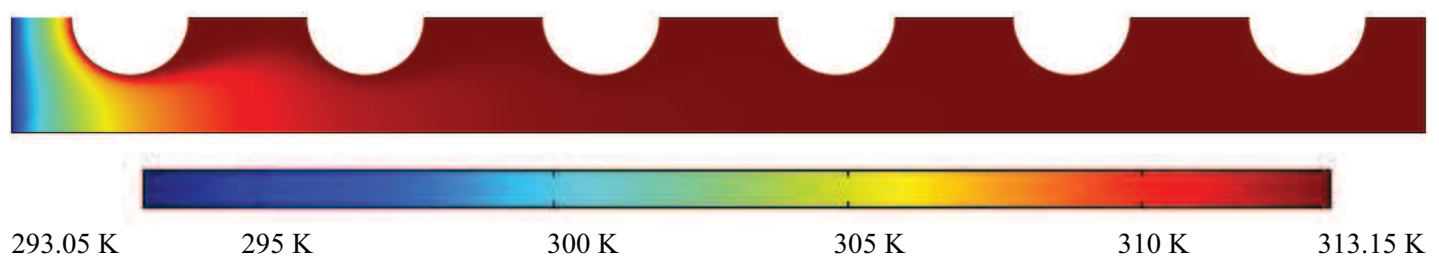

(c) Temperature contours.

Figure 6: Streamlines, isotherms and temperature contours for in-line configuration, $R e_{D}=$ $480, D=D_{b}$ and $\delta=\delta_{b}$. 


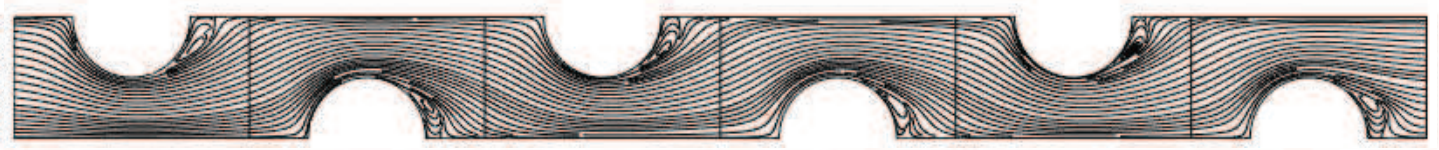

(a) Streamlines.

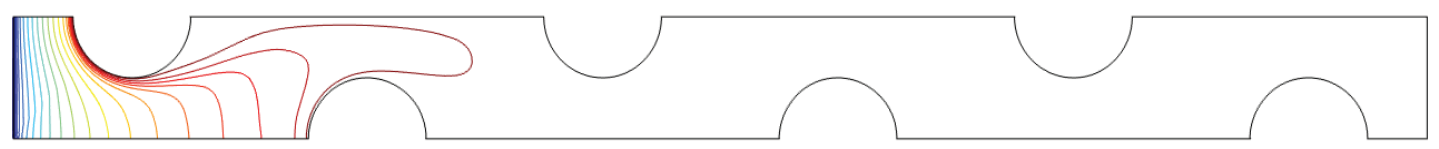

(b) Isotherms.

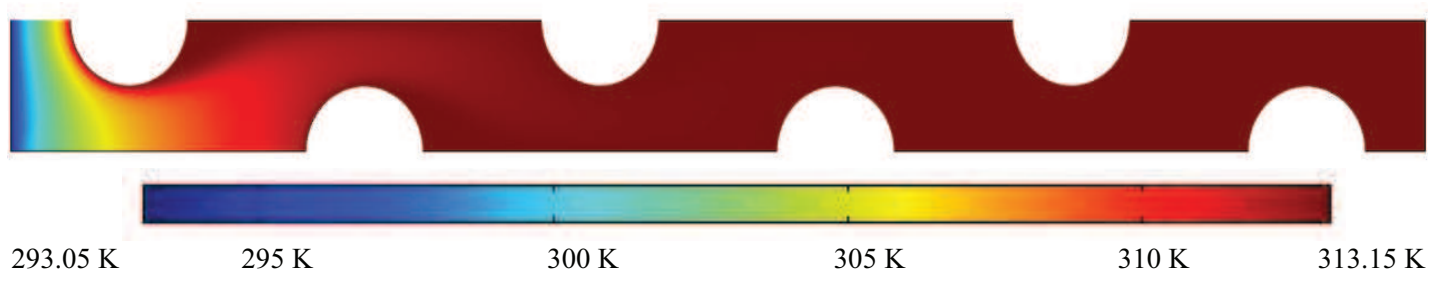

(c) Temperature contours.

Figure 7: Streamlines, isotherms and temperature contours for staggered configuration, $R e_{D}=480, D=D_{b}$ and $\delta=\delta_{b}$. 


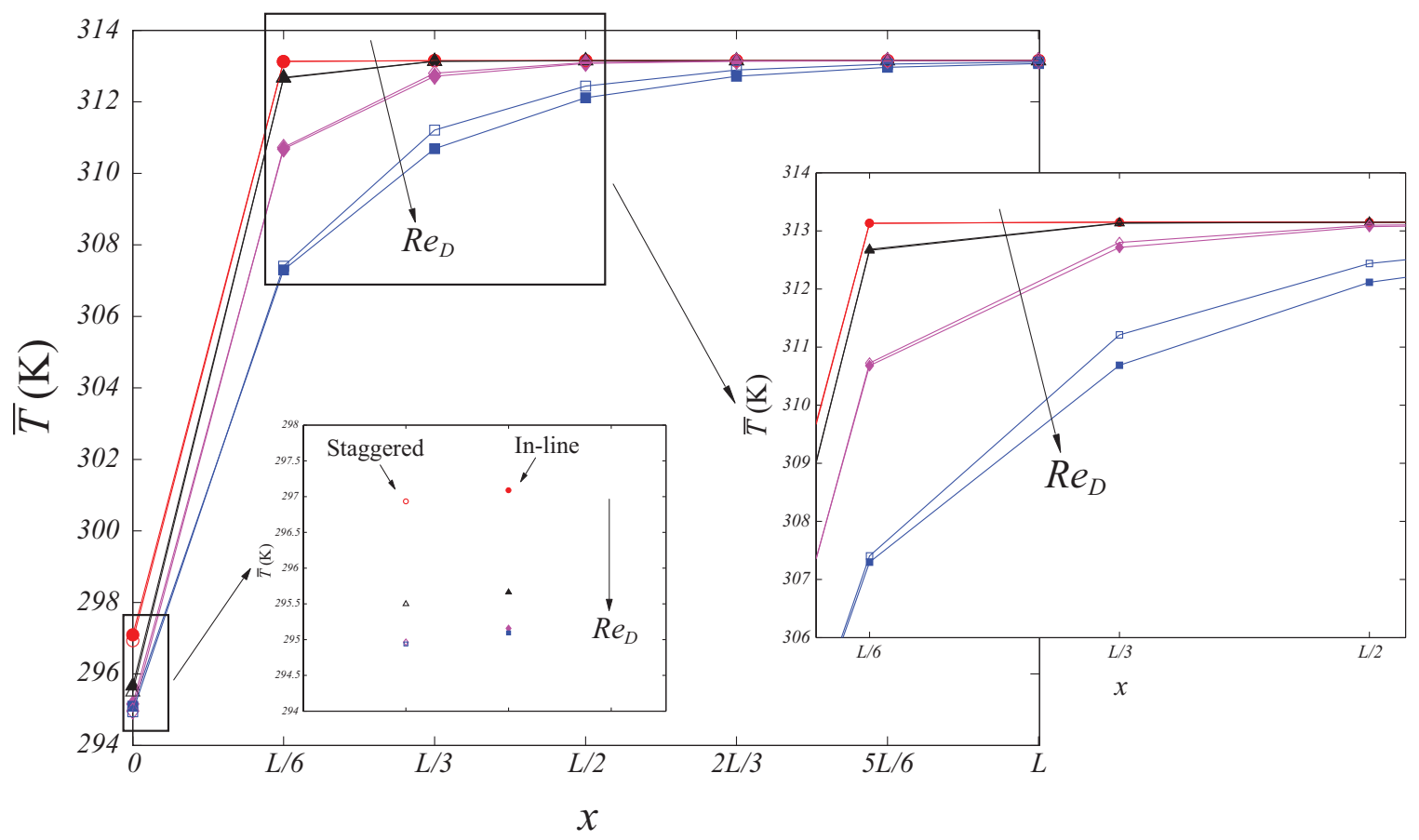

Figure 8: Streamwise distribution of $\bar{T}$ for $D=D_{b}, \delta=\delta_{b}$ and different $R e_{D}$. (- $\left.\bullet-,-\circ-\right)$ $R e_{D}=120 ;(-\boldsymbol{\Delta}-,-\triangle-) R e_{D}=240 ;\left(-\nabla_{-},-\diamond-\right) R e=480 ;(-\mathbf{\square}-,-\square-) R e=960$. Solid symbols are for in-line and open symbols are for staggered configurations, respectively. The symbols showing the in-line and staggered averaged-temperatures at the inlet $(x=0)$ of the heat exchanger have been shifted horizontally for clarity. 


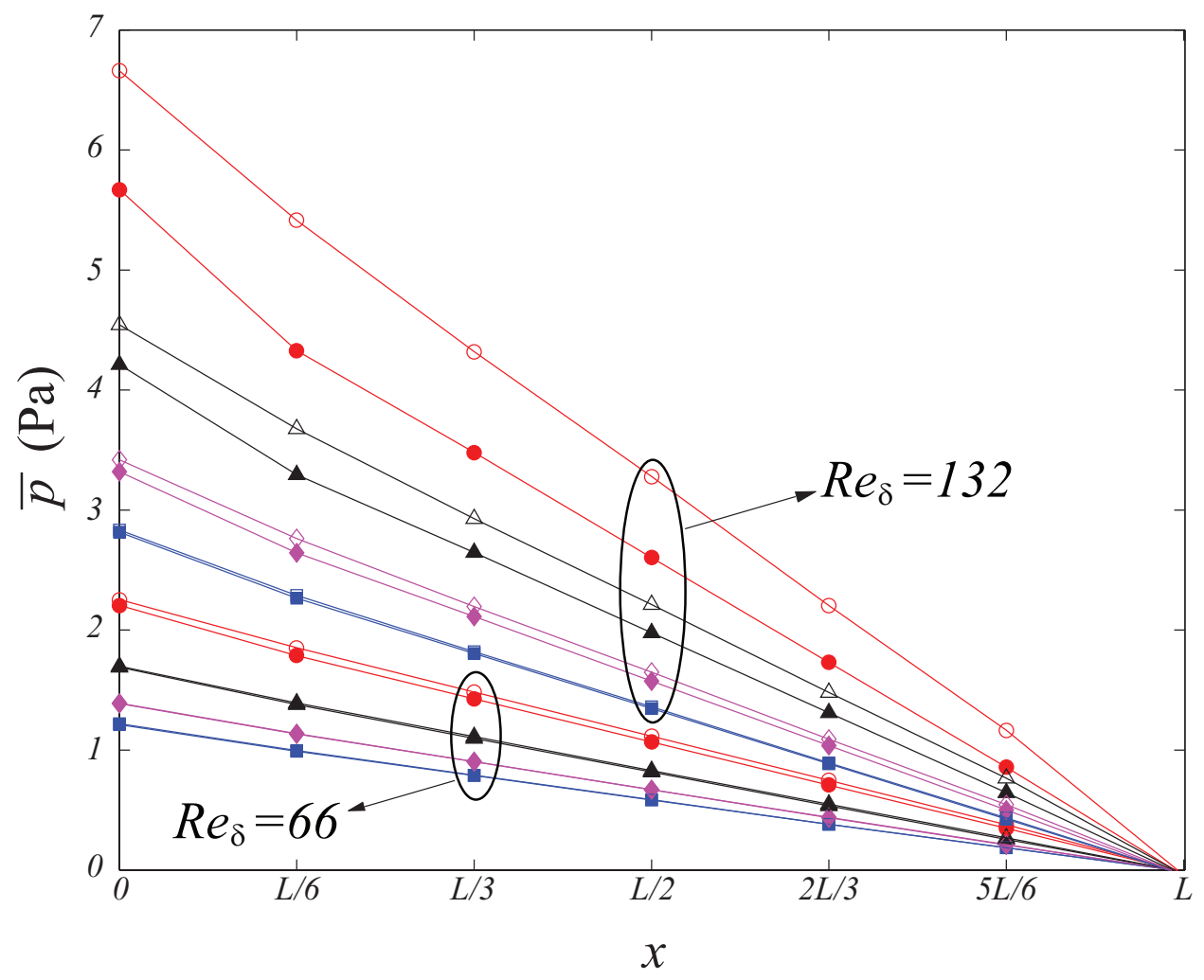

Figure 9: Streamwise distribution of $\bar{p}$ for $\delta=\delta_{b}, R e_{\delta}=66$ and $R e_{\delta}=132$, and different tube diameters. $(-\mathbf{\square}-,-\square-) D=0.25 D_{b} ;\left(-\nabla_{-},-\diamond-\right) D=0.5 D_{b} ;(-\boldsymbol{\Delta}-,-\triangle-) D=0.75 D_{b}$; $(-\bullet-,-\circ-) D=D_{b}$. Solid symbols are for in-line and open symbols are for staggered configurations, respectively. 


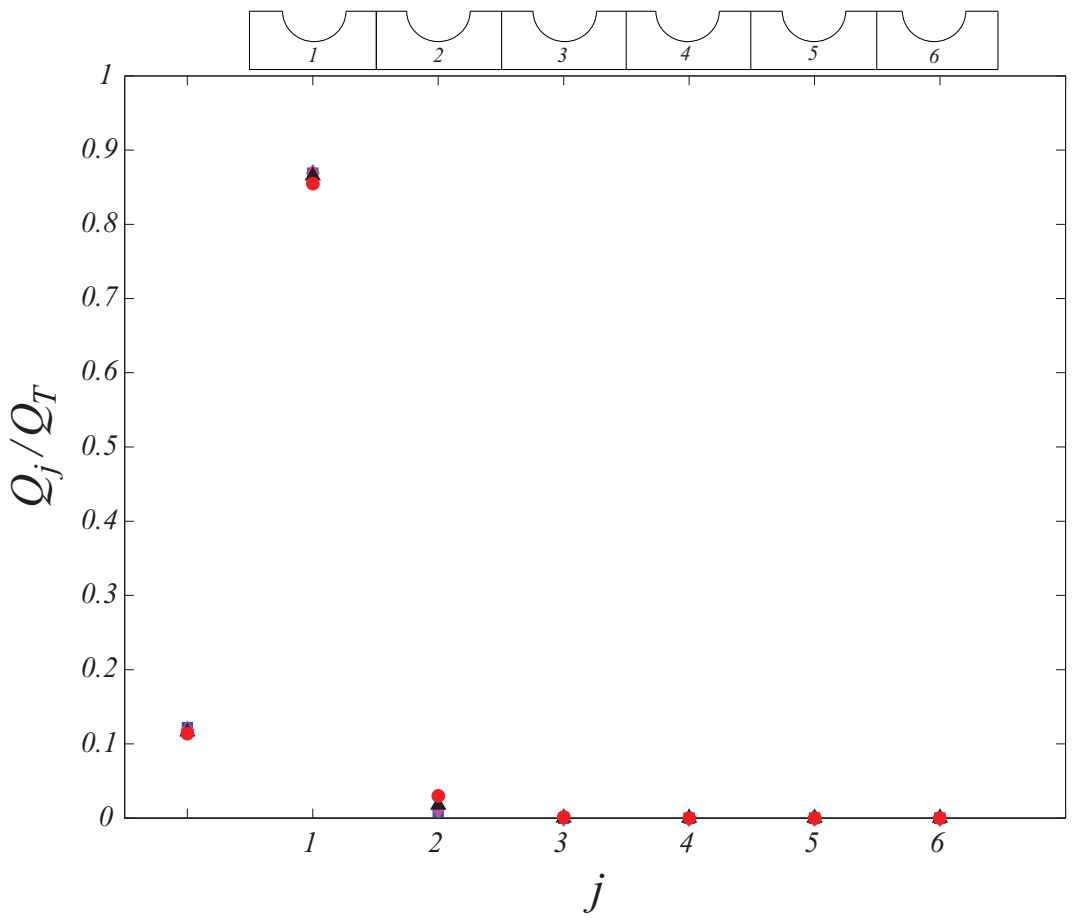

(a) In-line configuration.

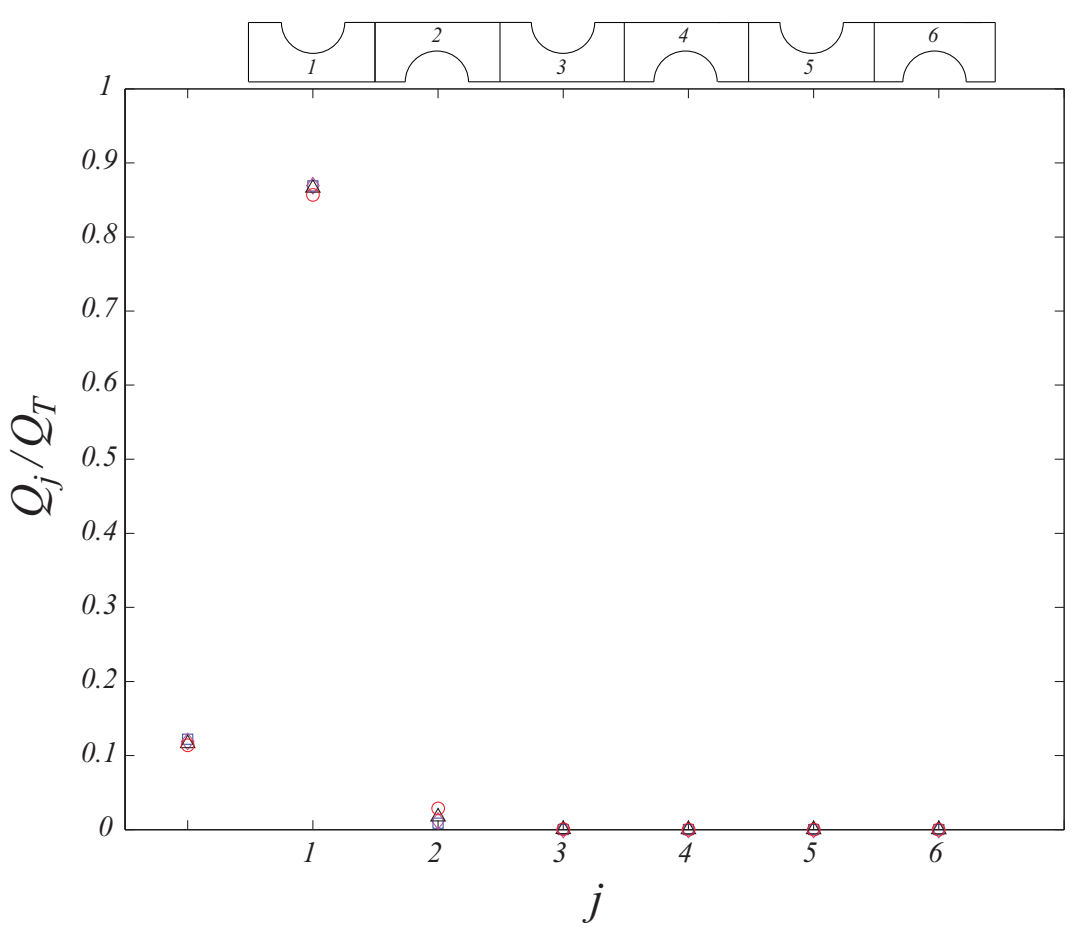

(b) Staggered configuration.

Figure 10: Fraction of heat rate $Q_{j} / Q_{T}$ for $R e_{\delta}=33, \delta=\delta_{b}$ and different values of $D$. $(\boldsymbol{\square}, \square) D=0.25 D_{b} ;(\diamond, \diamond) D=0.5 D_{b} ;(\boldsymbol{\Lambda}, \triangle) D=0.75 D_{b} ;(\bullet, \circ) D=D_{b}$. 


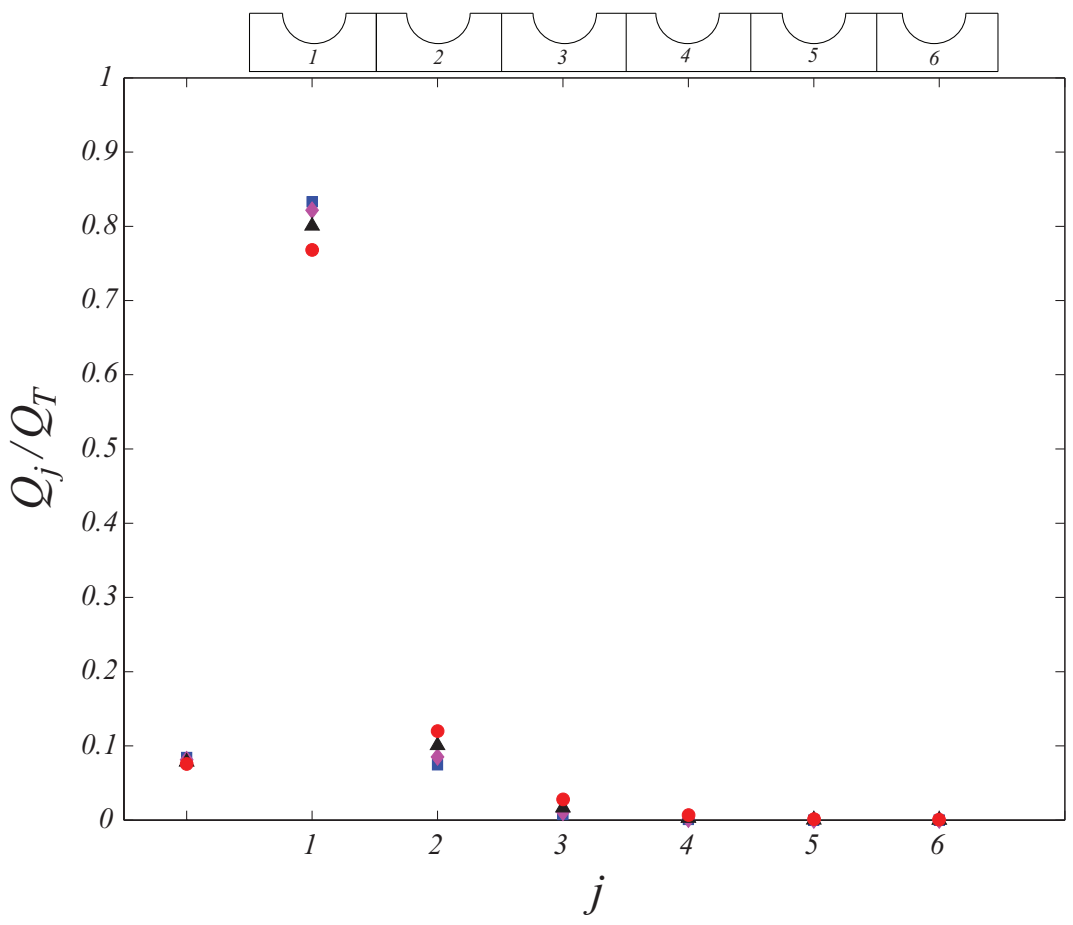

(a) In-line configuration.

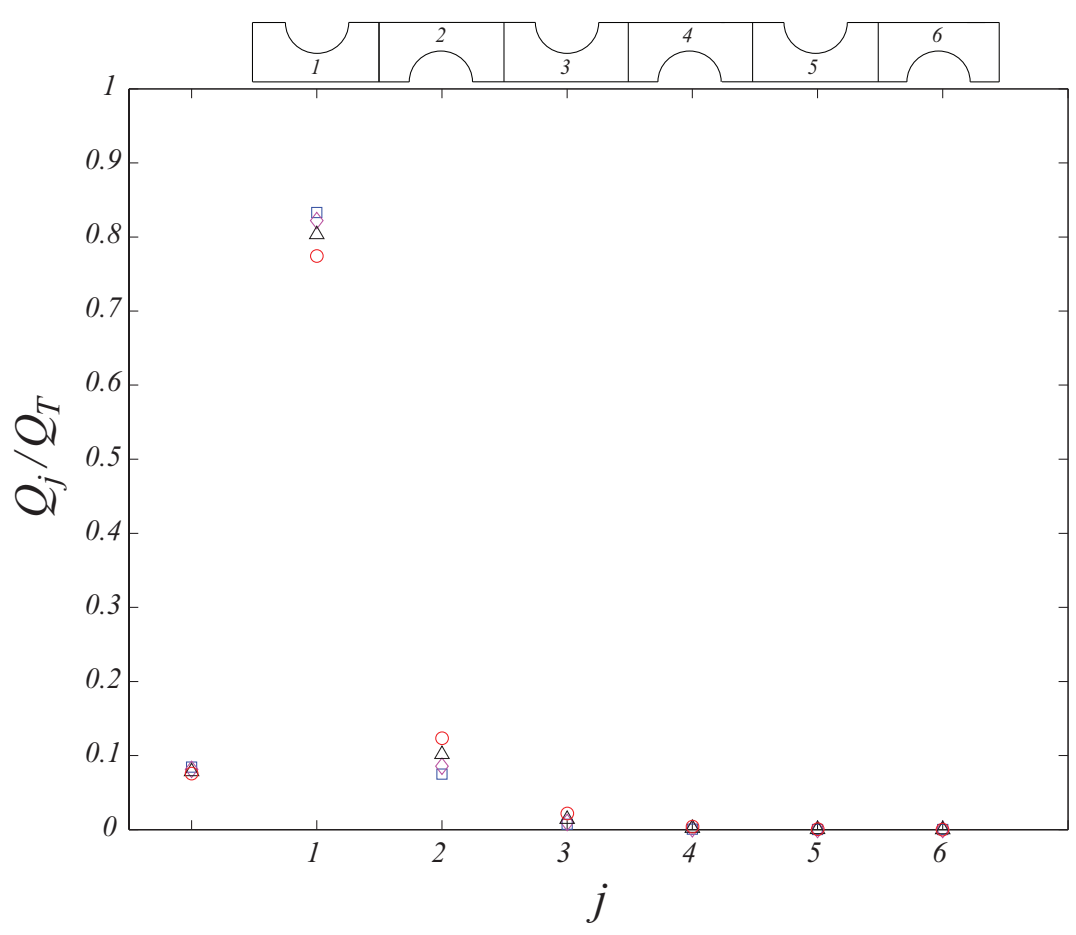

(b) Staggered configuration.

Figure 11: Fraction of heat rate $Q_{j} / Q_{T}$ for $R e_{\delta}=66, \delta=\delta_{b}$ and different values of $D$. $(\boldsymbol{\square}, \square) D=0.25 D_{b} ;(\diamond) D=0.5 D_{b} ;(\boldsymbol{\Lambda}, \triangle) D=0.75 D_{b} ;(\bullet, \circ) D=D_{b}$. 


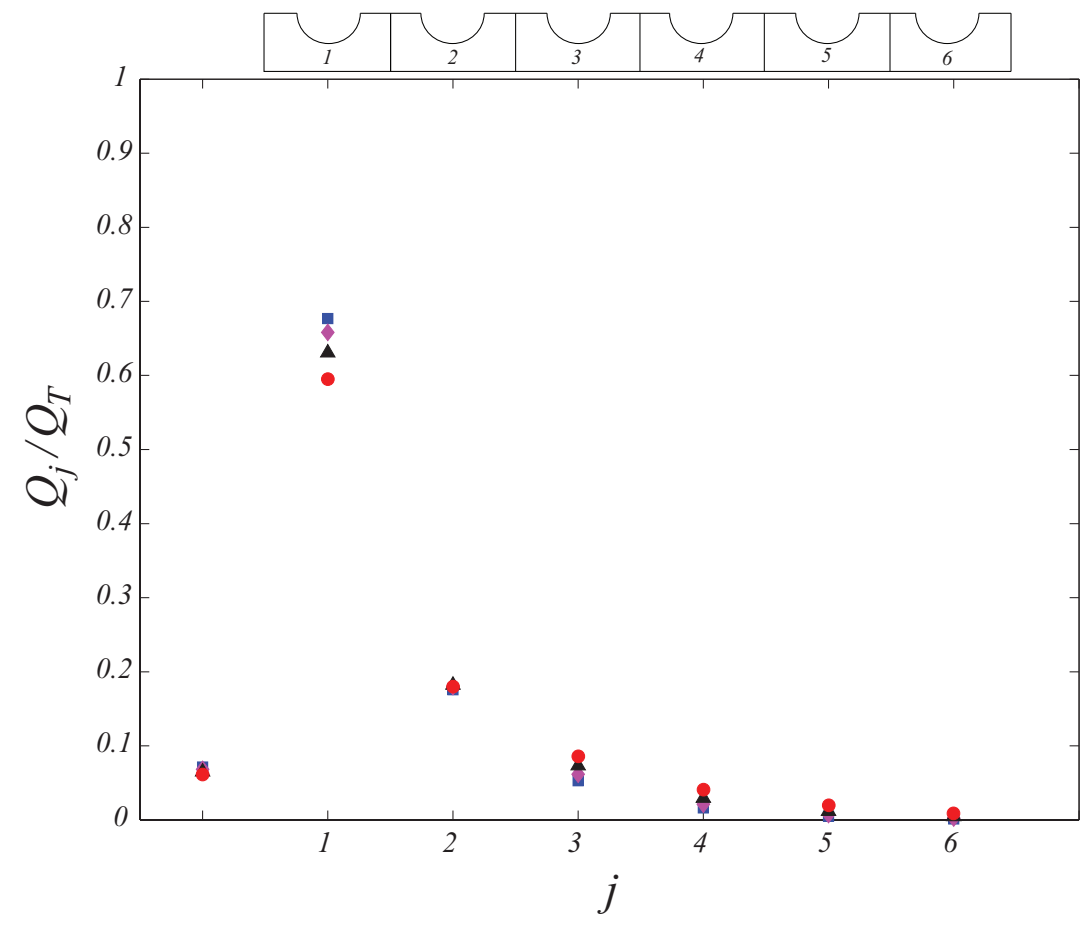

(a) In-line configuration.

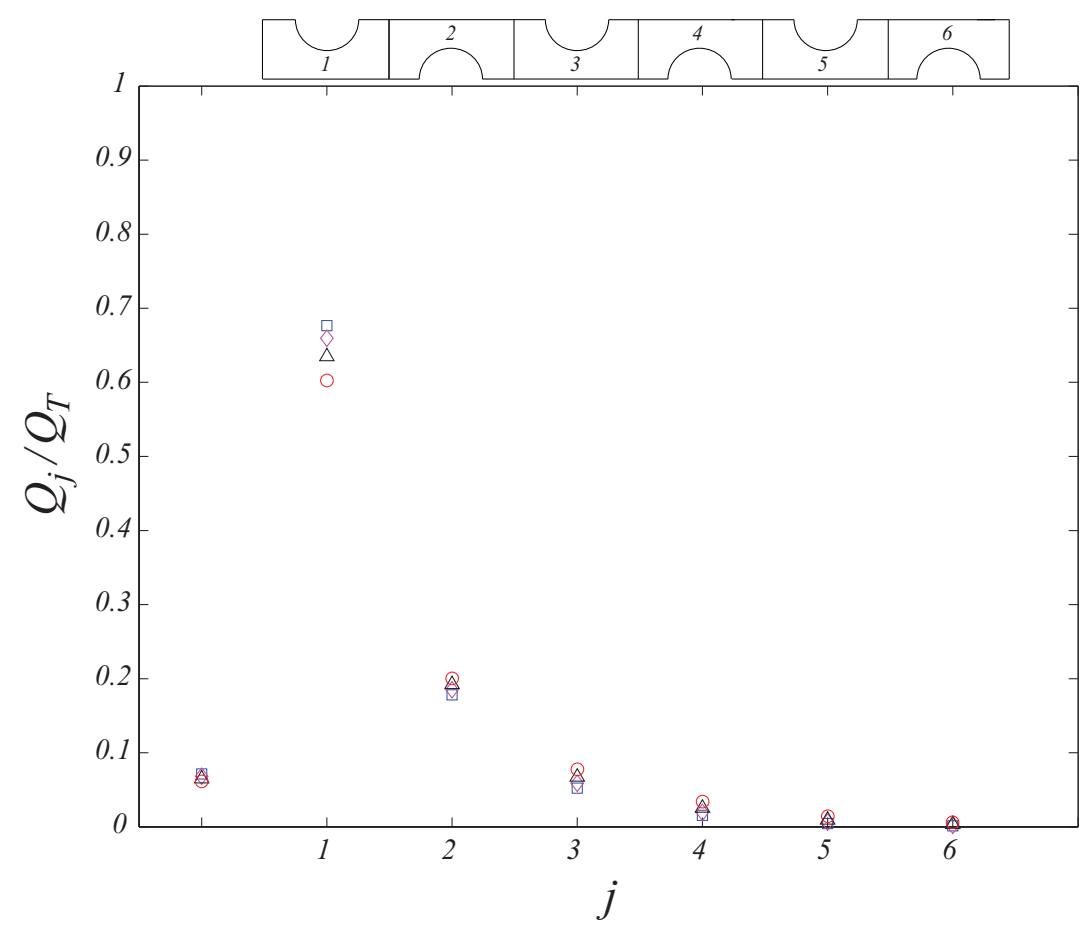

(b) Staggered configuration.

Figure 12: Fraction of heat rate $Q_{j} / Q_{T}$ for $\operatorname{Re}_{\delta}=132, \delta=\delta_{b}$ and different values of $D$. $(\boldsymbol{\square}, \square) D=0.25 D_{b} ;(\diamond, \diamond) D=0.5 D_{b} ;(\boldsymbol{\Lambda}, \triangle) D=0.75 D_{b} ;(\bullet, \circ) D=D_{b}$. 


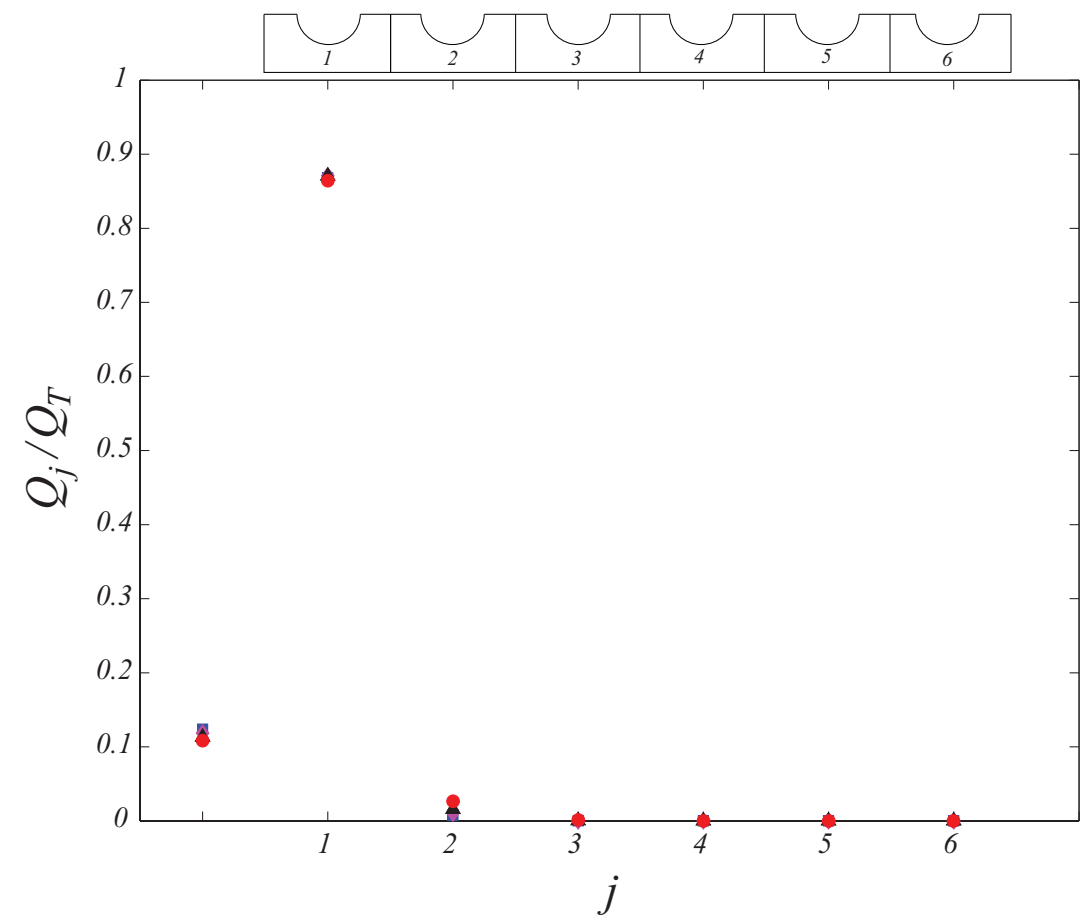

(a) In-line configuration.

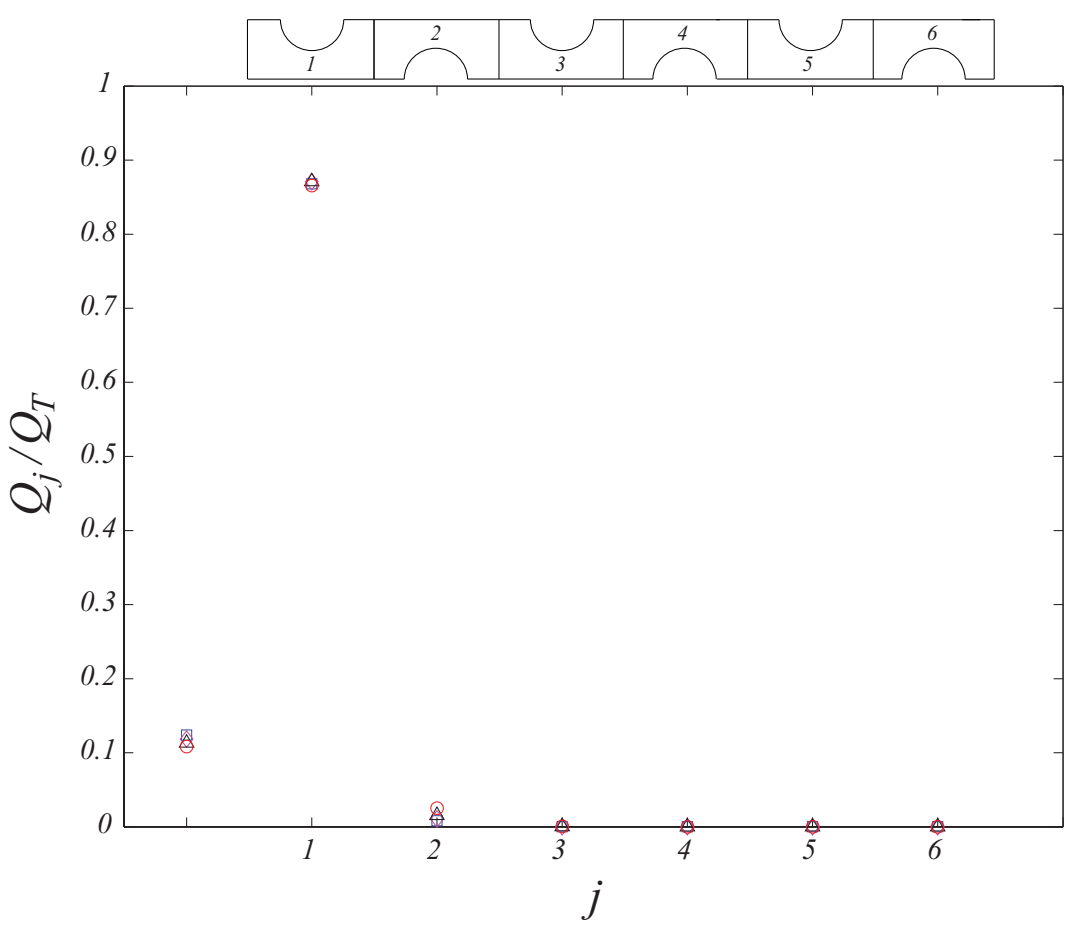

(b) Staggered configuration.

Figure 13: Fraction of heat rate $Q_{j} / Q_{T}$ for $R e_{D}=960, \delta=\delta_{b} / 2$ and different values of $D$. $\left.(\boldsymbol{\square}, \square) D=0.25 D_{b} ;(\diamond, \diamond) D=0.5 D_{b} ;(\boldsymbol{\Lambda}, \triangle) D=0.75 D_{b} ; \bullet \bullet, \circ\right) D=D_{b}$. 